\title{
A New Global Regression Analysis Method for the Prediction of Wind Tunnel Model Weight Corrections
}

\author{
N. Ulbrich ${ }^{\dagger}$ and T. Bridge $\ddagger$ \\ Jacobs Technology Inc., Moffett Field, California 94035-1000 \\ M. Amaya \\ NASA Ames Research Center, Moffett Field, California 94035-1000
}

\begin{abstract}
A new global regression analysis method is discussed that predicts wind tunnel model weight corrections for strain-gage balance loads during a wind tunnel test. The method determines corrections by combining "wind-on" model attitude measurements with least squares estimates of the model weight and center of gravity coordinates that are obtained from "wind-off" data points. The method treats the least squares fit of the model weight separate from the fit of the center of gravity coordinates. Therefore, it performs two fits of "windoff" data points and uses the least squares estimator of the model weight as an input for the fit of the center of gravity coordinates. Explicit equations for the least squares estimators of the weight and center of gravity coordinates are derived that simplify the implementation of the method in the data system software of a wind tunnel. In addition, recommendations for sets of "wind-off" data points are made that take typical model support system constraints into account. Explicit equations of the confidence intervals on the model weight and center of gravity coordinates and two different error analyses of the model weight prediction are also discussed in the appendices of the paper.
\end{abstract}

\section{Nomenclature}

$\begin{array}{ll}\widehat{\mathbf{A}} & =\text { matrix containing regressors of the first least squares problem } \\ \widehat{\mathbf{A}} & =\text { matrix containing regressors of the second least squares problem } \\ A F & =\text { axial force of the alternate balance axis system (see Fig. } 5 \text { or Ref. [1], Fig. 1) } \\ A F_{\text {weight }} & =\text { weight correction to the axial force of the alternate balance axis system (see Fig. } 5 \text { ) } \\ A_{\text {aero }} & =\text { axial force that is caused by aerodynamic loads on the wind tunnel model } \\ A_{\text {bal }} & =\text { axial force that acts in the primary balance axis system (see Fig. } 2 \text { ) } \\ A_{\text {model }} & =\text { axial force that is caused by the weight of the wind tunnel model } \\ A_{\text {sleeve }} & =\text { axial force that is caused by the weight of the sleeve (metric part of the balance) } \\ A_{\text {weight }} & =\text { axial force that is caused by the combined weight of the model and the sleeve } \\ \overline{\mathbf{B}} & =\text { vector containing the dependent variables of the first least squares problem } \\ \widehat{\mathbf{B}} & =\text { vector containing the dependent variables of the second least squares problem } \\ \mathbf{D} & =\text { components of vector } \mathbf{D} \text { in the primary balance axis system } \\ d_{x}, d_{y}, d_{z} & =\text { least squares estimators of } d_{x}, d_{y}, d_{z} \\ \widehat{d_{x}}, \widehat{d}_{y}, \widehat{d}_{z} & =\text { set of unit basis vectors of the primary or alternate balance axis system } \\ \mathbf{e}_{\mathbf{x}}, \mathbf{e}_{\mathbf{y}}, \mathbf{e}_{\mathbf{z}} & =\text { gravity vector in the primary balance axis system } \\ \mathbf{G} & =\text { components of the gravity vector } \mathbf{G} \text { in the primary balance axis system } \\ g_{x}, g_{y}, g_{z} & \left.=\text { gravitational acceleration (equals } 9.81\left[m / s^{2}\right] \text { or } 32.17 \text { [ } f t / s^{2}\right] \text { at sea level) } \\ g & =\text { index of an observation }\end{array}$

$\dagger$ Aerodynamicist, Jacobs Technology Inc.

\$ Software Developer, Jacobs Technology Inc.

$\S$ Data Engineer, NASA Ames Research Center 


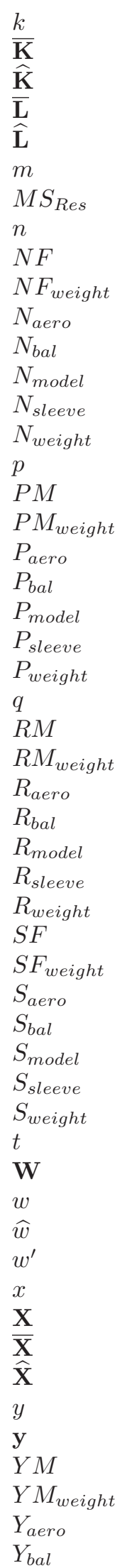

$=$ number of regressors used in Ref. [4]

$=$ auxiliary matrix used by the first least squares problem

$=$ auxiliary matrix used by the second least squares problem

$=$ auxiliary vector used by the first least squares problem

$=$ auxiliary vector used by the second least squares problem

$=$ combined mass of the wind tunnel model and the metric part of the balance

$=$ residual mean square

$=$ total number of observations

$=$ normal force of the alternate balance axis system (see Fig. 5 or Ref. [1], Fig. 1)

$=$ weight correction to the normal force of the alternate balance axis system (see Fig. 5)

$=$ normal force that is caused by aerodynamic loads on the wind tunnel model

$=$ normal force that acts in the primary balance axis system (see Fig. 2)

$=$ normal force that is caused by the weight of the wind tunnel model

$=$ normal force that is caused by the weight of the sleeve (metric part of the balance)

$=$ normal force that is caused by the combined weight of the model and the sleeve

$=$ total number of regression model parameter

$=$ pitching moment of the alternate balance axis system (see Fig. 5 or Ref. [1], Fig. 1)

$=$ weight correction to the pitching moment of the alternate balance axis system (see Fig. 5)

$=$ pitching moment that is caused by aerodynamic loads on the wind tunnel model

$=$ pitching moment that acts in the primary balance axis system (Fig. 2)

$=$ pitching moment that is caused by the weight of the wind tunnel model

$=$ pitching moment that is caused by the weight of the sleeve (metric part of the balance)

$=$ pitching moment that is caused by the combined weight of the model and the sleeve

$=$ total number of data points used to fit the weight and center of gravity coordinates

$=$ rolling moment of the alternate balance axis system (see Fig. 5 or Ref. [1], Fig. 1)

$=$ weight correction to the rolling moment of the alternate balance axis system (see Fig. 5)

$=$ rolling moment that is caused by aerodynamic loads on the wind tunnel model

$=$ rolling moment that acts in the primary balance axis system (Fig. 2)

$=$ rolling moment that is caused by the weight of the wind tunnel model

$=$ rolling moment that is caused by the weight of the sleeve (metric part of the balance)

$=$ rolling moment that is caused by the combined weight of the model and the sleeve

$=$ side force of the alternate balance axis system (see Fig. 5 or Ref. [1], Fig. 1)

$=$ weight correction to the side force of the alternate balance axis system (see Fig. 5)

$=$ side force that is caused by aerodynamic loads on the wind tunnel model

$=$ side force that acts in the primary balance axis system (Fig. 2)

$=$ side force that is caused by the weight of the wind tunnel model

$=$ side force that is caused by the weight of the sleeve (metric part of the balance)

$=$ side force that is caused by the combined weight of the model and the sleeve

$=$ percentage point of the $t$-distribution (see, e.g., Ref. [4], p. 515, Table A.3)

$=$ weight vector, i.e., the force of gravity on a body

$=$ length of the weight vector $\mathbf{W}$; equals the "scalar" weight of the wind tunnel model

$=$ least squares estimator of $w$

$=$ weight estimate of a single data point

$=$ coordinate that defines the direction of the axial force - or- regressor variable

$=$ generic matrix that holds the regressor values of a multivariate least squares problem

$=$ vector containing the coefficients of the first least squares problem

$=$ vector containing the coefficients of the second least squares problem

$=$ coordinate that defines the direction of the side force - or - response variable

$=$ vector that holds the response values of a multivariate least squares problem

= yawing moment of the alternate balance axis system (see Fig. 5 or Ref. [1], Fig. 1)

$=$ weight correction to the yawing moment of the alternate balance axis system (see Fig. 5)

= yawing moment that is caused by aerodynamic loads on the wind tunnel model

= yawing moment that acts in the primary balance axis system (Fig. 2) 


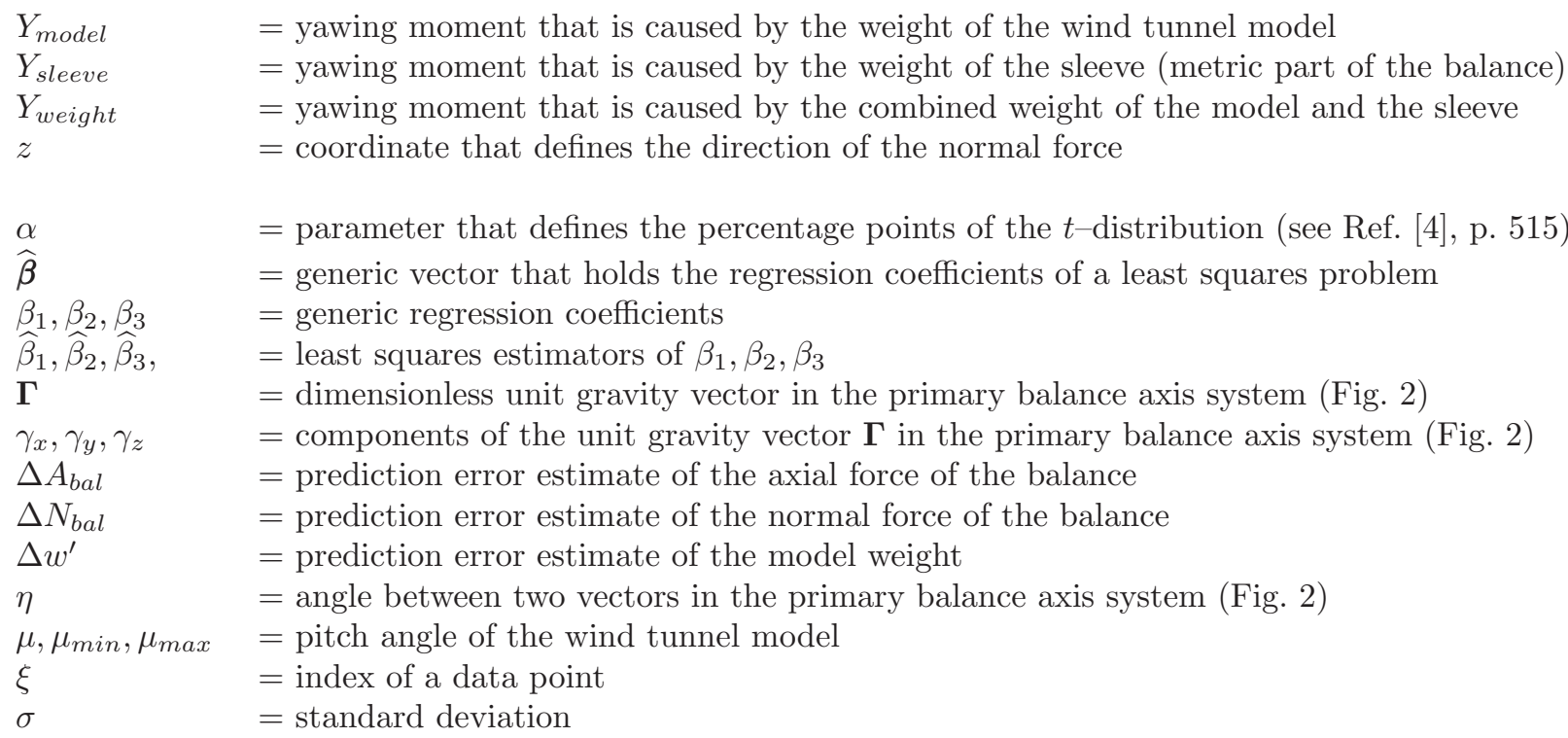

\section{Introduction}

A strain-gage balance is used during a wind tunnel test to measure forces and moments that act on a wind tunnel model. These loads can be determined, for example, in three steps. First, the raw gage outputs of the balance are measured as absolute voltages when the loads act on the wind tunnel model. Then, the natural zeros, i.e., the known gage outputs of the absolute load datum of the balance, are subtracted from the raw outputs to get the output changes relative to the absolute load datum of the balance. Finally, the output changes are used as input for a load iteration scheme that computes the balance loads (see Ref. [1] and [2] for a detailed description of the load iteration scheme).

The user of an "unpowered" wind tunnel model is primarily interested in aerodynamic loads that act on the model in a "wind-on" test condition. Unfortunately, a balance cannot directly distinguish between the part of its electrical outputs that is caused by aerodynamic loads and the part that is caused by the weight of the model (see Fig. 1). Therefore, the predicted balance load, i.e., the result of the load iterations, is always the sum of an aerodynamic load and a load that is caused by the weight of the model. This situation can be expressed by using the following relationships for the forces and moments of a six-component balance (the subscript "bal" marks the total predicted load; the subscript "aero" marks the part that is caused by aerodynamic loads; the subscript "weight" marks the part that is caused by the total model weight):

\section{"WIND-ON" TEST CONDITION}

$$
\begin{aligned}
A_{\text {bal }} & =A_{\text {aero }}+A_{\text {weight }} \\
S_{\text {bal }} & =S_{\text {aero }}+S_{\text {weight }} \\
N_{\text {bal }} & =N_{\text {aero }}+N_{\text {weight }} \\
R_{\text {bal }} & =R_{\text {aero }}+R_{\text {weight }} \\
P_{\text {bal }} & =P_{\text {aero }}+P_{\text {weight }} \\
Y_{\text {bal }} & =Y_{\text {aero }}+Y_{\text {weight }}
\end{aligned}
$$


It was mentioned earlier that the change of the strain-gage outputs relative to the natural zeros of the balance is used as input for the iterative balance load calculation because the natural zeros represent the outputs of the balance gages in an assumed "weightless" condition. Therefore, balance loads associated with weight effects are not just caused by the weight of the wind tunnel model. The weight of the balance sleeve, i.e., of the metric part of the balance, also has to be considered for the sake of completeness even though its contribution is small when compared with the weight of a typical wind tunnel model. Consequently, the total weight effects on the balance load measurements of a "wind-on" data point can be expressed by the following equations (the subscript "weight" marks the total model weight; the subscript "model" marks the part of the total model weight that is exclusively caused by the wind tunnel model; the subscript "sleeve" marks the part of the total model weight that is caused by the metric part of the balance):

$$
\begin{array}{rlrlrl}
A_{\text {weight }}=A_{\text {model }}+A_{\text {sleeve }} & \text { where } & & \left|A_{\text {model }}\right| \gg\left|A_{\text {sleeve }}\right| \\
S_{\text {weight }}=S_{\text {model }}+S_{\text {sleeve }} & \text { where } & \left|S_{\text {model }}\right| \gg\left|S_{\text {sleeve }}\right| \\
N_{\text {weight }}=N_{\text {model }}+N_{\text {sleeve }} & \text { where } & & \left|N_{\text {model }}\right| \gg\left|N_{\text {sleeve }}\right| \\
R_{\text {weight }}=R_{\text {model }}+R_{\text {sleeve }} & \text { where } & \left|R_{\text {model }}\right| \gg\left|R_{\text {sleeve }}\right| \\
P_{\text {weight }}=P_{\text {model }}+P_{\text {sleeve }} & \text { where } & \left|P_{\text {model }}\right| \gg\left|P_{\text {sleeve }}\right| \\
Y_{\text {weight }}=Y_{\text {model }}+Y_{\text {sleeve }} & \text { where } & \left|Y_{\text {model }}\right| \gg\left|Y_{\text {sleeve }}\right|
\end{array}
$$

The term "model weight" is frequently used in subsequent sections of the paper. Its usage implicitly assumes that the weight of the metric part of the balance is a part of the model weight.

Equations $(1 a)$ to $(1 f)$ indicate that loads caused by the weight of the model, i.e., $A_{\text {weight }}, \cdots, Y_{\text {weight }}$, have to be subtracted from the computed "raw" balance loads, i.e., $A_{b a l}, \cdots, Y_{b a l}$, so that the desired aerodynamic loads, i.e., $A_{a e r o}, \cdots, Y_{a e r o}$, are obtained. Consequently, a process has to be defined that determines balance loads caused by the total model weight for every "wind-on" data point. The development of such a process starts with a "first principles" analysis of all loads that are associated with the gravitational acceleration (i.e., $A_{\text {weight }}, \cdots, Y_{\text {weight }}$ for a six-component balance). This analysis and the subsequent definition of loads associated with the model weight are described in the next section of the paper.

\section{Definition of Model Weight Corrections}

It is necessary to describe all balance load components that are associated with the gravitational acceleration so that wind tunnel model weight corrections can be predicted. First, a balance axis system needs to be defined before the loads can be described. It is assumed that (i) the balance axis system is a right-handed coordinate system and that (ii) both force and moment vectors follow the sign convention of the related Cartesian coordinates. Figure 2 shows the corresponding coordinate system. It is also called the "primary" balance axis system. This axis system and its load sign definitions are the basis for all derivations that are discussed in the paper. It also has the useful property that the cross-product operator of vector algebra can rigorously be applied during the derivation of the model weight corrections. At this point of the analysis it is important to explicitly mention that the signs of two of the six loads used in the "primary" balance axis system do not agree with load sign definitions that are commonly adopted in experimental aeronautics. This difference of the sign definitions, however, can easily be overcome by performing two simple load transformations (for more details see the discussion given in Section V of the present paper).

The balance loads associated with the gravitational acceleration are determined by (i) the combined mass of the wind tunnel model and the metric part of the balance and (ii) the location of the gravity vector in the balance axis system. The gravity vector $\mathbf{G}$ can be described using the components of the gravitational acceleration in the balance axis system. Then, we get:

$$
\mathbf{G}=\left[\begin{array}{l}
g_{x} \\
g_{y} \\
g_{z}
\end{array}\right]
$$

American Institute of Aeronautics and Astronautics 
The length of the gravity vector equals the gravitational acceleration itself at the given elevation of an observer. Therefore, we know that

$$
|\mathbf{G}|=g=\sqrt{g_{x}^{2}+g_{y}^{2}+g_{z}^{2}}
$$

where, for example, the gravitational acceleration at sea level equals

$$
g=9.81\left[\mathrm{~m} / \mathrm{sec}^{2}\right] \quad \ldots \text { or } \ldots \quad g=32.17\left[\mathrm{ft} / \mathrm{sec}^{2}\right]
$$

It is convenient to describe the gravity vector as a dimensionless quantity because the current analysis is primarily interested in a description of the direction of the gravity vector in the balance axis system. Consequently, the following equation is valid:

$$
\mathbf{G}=g \cdot \boldsymbol{\Gamma}=g \cdot\left[\begin{array}{l}
\gamma_{x} \\
\gamma_{y} \\
\gamma_{z}
\end{array}\right]
$$

where

$$
\begin{aligned}
\gamma_{x} & =\frac{g_{x}}{g} \\
\gamma_{y} & =\frac{g_{y}}{g} \\
\gamma_{z} & =\frac{g_{z}}{g}
\end{aligned}
$$

The length of the dimensionless gravity vector $\boldsymbol{\Gamma}$ is one because the following relationship is valid:

$$
|\boldsymbol{\Gamma}|=\sqrt{\gamma_{x}^{2}+\gamma_{y}^{2}+\gamma_{z}^{2}}=\sqrt{\frac{g_{x}{ }^{2}+g_{y}{ }^{2}+g_{z}^{2}}{g^{2}}}=\sqrt{\frac{g^{2}}{g^{2}}}=1.0
$$

In the next step it is necessary to connect the total mass of the wind tunnel model and the dimensionless unit gravity vector $\boldsymbol{\Gamma}$ to the weight vector $\mathbf{W}$ (see Ref. [3], p. 127, for a definition of the weight vector). We know from Newtonian Mechanics that

$$
\mathbf{W}=m \cdot \mathbf{G}=m \cdot g \cdot \boldsymbol{\Gamma}=m \cdot g \cdot\left[\begin{array}{c}
\gamma_{x} \\
\gamma_{y} \\
\gamma_{z}
\end{array}\right]
$$

Then, after introducing the model weight as a scalar quantity, we can write:

$$
w=m \cdot g
$$

Consequently, the weight vector can be expressed as follows:

$$
\mathbf{W}=w \cdot\left[\begin{array}{l}
\gamma_{x} \\
\gamma_{y} \\
\gamma_{z}
\end{array}\right]=\left[\begin{array}{c}
\gamma_{x} \cdot w \\
\gamma_{y} \cdot w \\
\gamma_{z} \cdot w
\end{array}\right]
$$

The components of the weight vector are identical with the axial force, side force, and normal force that are caused by the weight of the model. Therefore, we get:

$$
\mathbf{W}=\left[\begin{array}{c}
A_{\text {weight }} \\
S_{\text {weight }} \\
N_{\text {weight }}
\end{array}\right]
$$


Finally, after equating the right-hand side of Eq. (9b) with the right-hand side of Eq. (9a), we get for the forces that are caused by the model weight in the "primary" balance axis system the following relationships:

\section{FORCES CAUSED BY THE MODEL WEIGHT}

$$
\begin{aligned}
A_{\text {weight }} & =\gamma_{x} \cdot w \\
S_{\text {weight }} & =\gamma_{y} \cdot w \\
N_{\text {weight }} & =\gamma_{z} \cdot w
\end{aligned}
$$

The moments associated with the weight of the model can be obtained using a similar approach. These moments have to be described relative to the balance moment center as the center of gravity of the model is not necessarily identical with the balance moment center. The present analysis simply assumes that the origin of the balance axis system is the balance moment center. Then, the center of gravity of the model can be described by the using the following vector (see also Fig. 3):

$$
\mathbf{D}=\left[\begin{array}{l}
d_{x} \\
d_{y} \\
d_{z}
\end{array}\right]
$$

Vector $\mathbf{D}$ describes the model's lever arms in the balance axis system. The torque caused by the model's weight with respect to the balance moment center is defined by the cross-product of the "lever arm" vector $\mathbf{D}$ and the "weight" vector W (see also Ref. [3], p. 302). In addition, we know that the components of the torque are the rolling, pitching, and yawing moment. Therefore, we can write:

$$
\mathbf{D} \times \mathbf{W}=\left[\begin{array}{c}
R_{\text {weight }} \\
P_{\text {weight }} \\
Y_{\text {weight }}
\end{array}\right]
$$

where

$$
\mathbf{D} \times \mathbf{W}=\left[\begin{array}{l}
d_{x} \\
d_{y} \\
d_{z}
\end{array}\right] \times\left[\begin{array}{c}
A_{\text {weight }} \\
S_{\text {weight }} \\
N_{\text {weight }}
\end{array}\right]
$$

The cross-product defined in Eq. (12b) needs to be computed. In general, the cross-product of two vectors can be described as a determinate. Then, we get:

$$
\mathbf{D} \times \mathbf{W}=\left|\begin{array}{ccc}
\mathbf{e}_{\mathbf{x}} & \mathbf{e}_{\mathbf{y}} & \mathbf{e}_{\mathbf{z}} \\
d_{x} & d_{y} & d_{z} \\
A_{\text {weight }} & S_{\text {weight }} & N_{\text {weight }}
\end{array}\right|=\left[\begin{array}{c}
d_{y} \cdot N_{\text {weight }}-d_{z} \cdot S_{\text {weight }} \\
d_{z} \cdot A_{\text {weight }}-d_{x} \cdot N_{\text {weight }} \\
d_{x} \cdot S_{\text {weight }}-d_{y} \cdot A_{\text {weight }}
\end{array}\right]
$$

The forces $A_{\text {weight }}, S_{\text {weight }}$, and $N_{\text {weight }}$ used in Eq. (12c) can be replaced by the right-hand sides of Eqs. $(10 a),(10 b)$, and $(10 c)$. Then, Eq. (12c) becomes:

$$
\mathbf{D} \times \mathbf{W}=\left[\begin{array}{l}
\gamma_{z} \cdot w \cdot d_{y}-\gamma_{y} \cdot w \cdot d_{z} \\
\gamma_{x} \cdot w \cdot d_{z}-\gamma_{z} \cdot w \cdot d_{x} \\
\gamma_{y} \cdot w \cdot d_{x}-\gamma_{x} \cdot w \cdot d_{y}
\end{array}\right]
$$

In the last step, after equating the right-hand side of Eq. (13) with the right-hand side of Eq. (12a), we get for the moments in the "primary" balance axis system the following relationships: 


\section{MOMENTS CAUSED BY THE MODEL WEIGHT}

$$
\begin{aligned}
& R_{\text {weight }}=\gamma_{z} \cdot w \cdot d_{y}-\gamma_{y} \cdot w \cdot d_{z} \\
& P_{\text {weight }}=\gamma_{x} \cdot w \cdot d_{z}-\gamma_{z} \cdot w \cdot d_{x} \\
& Y_{\text {weight }}=\gamma_{y} \cdot w \cdot d_{x}-\gamma_{x} \cdot w \cdot d_{y}
\end{aligned}
$$

The six equations (10a), (10b), (10c), (14a), (14b), and (14c) define all forces and moments that are caused by the model weight. These loads are known for any "wind-on" data point if the following three sets of parameters are given: Set $1 \Longrightarrow$ the components $\gamma_{x}, \gamma_{y}, \gamma_{z}$ of the unit gravity vector of the "wind-on" data point in the balance axis system; $\underline{\text { Set } 2} \Longrightarrow$ the weight $w$ of the wind tunnel model; $\underline{\text { Set } 3} \Longrightarrow$ the center of gravity coordinates $d_{x}, d_{y}, d_{z}$ of the wind tunnel model in the balance axis system.

The components of the unit gravity vector change for each "wind-on" data point because any model attitude change also changes the components of the unit gravity vector in the balance axis system. Therefore, $\gamma_{x}, \gamma_{y}, \gamma_{z}$ have to be measured for each "wind-on" data point in "real-time" by using the wind tunnel's model attitude measurement process. The weight of the wind tunnel model $(w)$ and its center of gravity coordinates $\left(d_{x}, d_{y}, d_{z}\right)$, on the other hand, are four physical constants of the chosen model configuration as long as (i) no hardware is added to or removed from the model and (ii) the model's weight distribution is not changed. These four constants have to be determined experimentally for a "real-world" wind tunnel model because the complexity of its geometry and weight distribution makes an analytic determination impractical.

The four constants may be obtained, for example, by applying a regression analysis, i.e., a least squares fit, to a series of "wind-off" data points. This approach has two major advantages. First, only a regression analysis in combination with a suitable experimental design and a tight control over the most important measurement error sources can ensure that both systematic and random errors contained in numerical estimates of the model's weight and center of gravity coordinates are within a wind tunnel customer's tolerances. In addition, aerodynamic loads of "wind-off" data points are zero by design (see also Fig. 4). Consequently, the measured strain-gage outputs and predicted loads of "wind-off" data points are exclusively caused by model weight effects. This observation can be expressed by using the following set of equations

\begin{tabular}{|c|c|c|c|c|c|c|c|}
\hline \multicolumn{4}{|c|}{ "WIND-OFF } & \multicolumn{3}{|c|}{ TEST CONDITION } & \multirow[b]{2}{*}{$(15 a)$} \\
\hline$A_{\text {aero }}$ & $=$ & 0 & $\Longrightarrow$ & $A_{b a l}$ & $=$ & $A_{\text {weight }}$ & \\
\hline$S_{\text {aero }}$ & $=$ & 0 & $\Longrightarrow$ & $S_{b a l}$ & $=$ & $S_{\text {weight }}$ & $(15 b)$ \\
\hline$N_{\text {aero }}$ & $=$ & 0 & $\Longrightarrow$ & $N_{b a l}$ & $=$ & $N_{\text {weight }}$ & $(15 c)$ \\
\hline$R_{\text {aero }}$ & $=$ & 0 & $\Longrightarrow$ & $R_{b a l}$ & $=$ & $R_{\text {weight }}$ & $(15 d)$ \\
\hline$P_{\text {aero }}$ & $=$ & 0 & $\Longrightarrow$ & $P_{b a l}$ & $=$ & $P_{\text {weight }}$ & $(15 e)$ \\
\hline$Y_{\text {aero }}$ & $=$ & 0 & $\Longrightarrow$ & $Y_{b a l}$ & $=$ & $Y_{\text {weight }}$ & $(15 f)$ \\
\hline
\end{tabular}
(the subscript "bal" marks the total load; the subscript "aero" marks the part that is caused by aerodynamic loads; the subscript "weight" marks the part that is caused by the total model weight):

The calculation of least squares estimators of the weight and the center of gravity coordinates of the wind tunnel model is described in more detail in the next section of the paper. 


\section{Global Regression Analysis}

\section{A. General Remarks}

A new method is derived in this section that may be used to determine least squares estimators of the weight $(w)$ and center of gravity coordinates $\left(d_{x}, d_{y}, d_{z}\right)$ of a wind tunnel model by exclusively using $\underline{\text { explicit }}$ equations. This novel approach greatly simplifies the implementation of the method in the data system of a wind tunnel facility as complexities associated with the coding of a global regression analysis algorithm are avoided. In principle, the method performs a global regression analysis of a set of "wind-off" data points. It makes the assumption that the least squares fit of the weight of the model can be separated from the least squares fit of the center of gravity coordinates. In other words, the new method performs two "coupled" least squares fits of "wind-off" data points as it uses the result of the first least squares fit, i.e., the least squares estimator of the model weight, as an input for the second least squares fit.

The global regression analysis of the set of "wind-off" data points is explained in detail in the next sections of the paper. More general information about the theoretical background of the global regression analysis approach can be found in the literature (see, e.g., Refs. [4], [5], [6], and [7] for more details about global regression analysis).

\section{B. Least Squares Estimator of the Model Weight}

First, the new method fits the model weight by exclusively using the three force components that are measured for each "wind-off" data point. Index $\xi$ describes an individual "wind-off" data point assuming that a total number of $q$ data points were recorded. Then, after applying Eqs. (10a) to (10c), we get:

$$
\begin{aligned}
& A_{\text {bal }}(\xi)=\gamma_{x}(\xi) \cdot \underbrace{w}_{\text {unknown }} ; 1 \leq \xi \leq q \\
& S_{\text {bal }}(\xi)=\gamma_{y}(\xi) \cdot \underbrace{w}_{\text {unknown }} ; \quad 1 \leq \xi \leq q \\
& N_{\text {bal }}(\xi)=\gamma_{z}(\xi) \cdot \underbrace{w}_{\text {unknown }} ; 1 \leq \xi \leq q
\end{aligned}
$$

Equations $(16 a)$ to $(16 c)$ can be summarized in vector format. Then, we get:

$$
\underbrace{\left[\begin{array}{c}
\gamma_{x}(\xi) \\
\gamma_{y}(\xi) \\
\gamma_{z}(\xi)
\end{array}\right]}_{\text {model attitude }} \cdot \underbrace{w}_{\text {unknown }}=\underbrace{\left[\begin{array}{c}
A_{\text {bal }}(\xi) \\
S_{\text {bal }}(\xi) \\
N_{\text {bal }}(\xi)
\end{array}\right]}_{\text {balance load }}
$$

Now, a more simple notation of the above two vectors is introduced. In addition, the model weight $w$ is replaced by its least squares estimator $\widehat{w}$. Then, we get:

$$
\begin{aligned}
\overline{\mathbf{K}}(\xi)_{3 \times 1} & =\left[\begin{array}{c}
\gamma_{x}(\xi) \\
\gamma_{y}(\xi) \\
\gamma_{z}(\xi)
\end{array}\right] \\
\overline{\mathbf{L}}(\xi)_{3 \times 1} & =\left[\begin{array}{c}
A_{b a l}(\xi) \\
S_{b a l}(\xi) \\
N_{b a l}(\xi)
\end{array}\right] \\
\overline{\mathbf{X}}_{1 \times 1} & =\widehat{w}
\end{aligned}
$$

Then, Eq. (17) can be expressed as follows for a single data point with index $\xi$ :

$$
\overline{\mathbf{K}}(\xi)_{3 \times 1} \cdot \overline{\mathbf{X}}_{1 \times 1}=\overline{\mathbf{L}}(\xi)_{3 \times 1}
$$


Consequently, we get the following equation if Eq. (19a) is expanded to all "wind-off" data points:

$$
\left[\begin{array}{c}
\overline{\mathbf{K}}(1)_{3 \times 1} \\
\overline{\mathbf{K}}(2)_{3 \times 1} \\
\cdots \\
\overline{\mathbf{K}}(q)_{3 \times 1}
\end{array}\right] \cdot \overline{\mathbf{X}}_{1 \times 1}=\left[\begin{array}{c}
\overline{\mathbf{L}}(1)_{3 \times 1} \\
\overline{\mathbf{L}}(2)_{3 \times 1} \\
\cdots \\
\overline{\mathbf{L}}(q)_{3 \times 1}
\end{array}\right]
$$

For convenience, the two vectors associated with all data points can be abbreviated as follows:

$$
\begin{aligned}
\overline{\mathbf{A}}_{3 q \times 1} & =\left[\begin{array}{c}
\overline{\mathbf{K}}(1)_{3 \times 1} \\
\overline{\mathbf{K}}(2)_{3 \times 1} \\
\cdots \\
\overline{\mathbf{K}}(q)_{3 \times 1}
\end{array}\right] \\
\overline{\mathbf{B}}_{3 q \times 1} & =\left[\begin{array}{c}
\overline{\mathbf{L}}(1)_{3 \times 1} \\
\overline{\mathbf{L}}(2)_{3 \times 1} \\
\cdots \\
\overline{\mathbf{L}}(q)_{3 \times 1}
\end{array}\right]
\end{aligned}
$$

Then, after using Eqs. (20a) and (20b) to simplify Eq. (19b), the equations of the first global regression analysis problem of the new method are obtained. We get:

$$
\text { First Global Regression Analysis Problem } \Longrightarrow \overline{\mathbf{A}}_{3 q \times 1} \cdot \overline{\mathbf{X}}_{1 \times 1}=\overline{\mathbf{B}}_{3 q \times 1}
$$

The solution of the first global regression problem, i.e., the least squares estimator of the model weight, is simply given by the following matrix equation:

\section{LEAST SQUARES ESTIMATOR OF THE MODEL WEIGHT}

$$
\overline{\mathbf{X}}_{1 \times 1}=\widehat{w}=\left(\overline{\mathbf{A}}^{\mathbf{T}} \cdot \overline{\mathbf{A}}\right)_{1 \times 1}^{-1} \cdot\left(\overline{\mathbf{A}}^{\mathbf{T}} \cdot \overline{\mathbf{B}}\right)_{1 \times 1}
$$

The right-hand side of Eq. (22) is the new method's least squares estimator of the model weight. It is possible to derive an explicit formula for the model weight after recognizing that the right-hand side of Eq. (22) is simply the product of two scalars. Therefore, by inspection, we can write:

$$
\begin{aligned}
\left(\overline{\mathbf{A}}^{\mathbf{T}} \cdot \overline{\mathbf{A}}\right)_{1 \times 1} & =\sum_{\xi=1}^{q} \underbrace{\left[\gamma_{x}^{2}(\xi)+\gamma_{y}^{2}(\xi)+\gamma_{z}^{2}(\xi)\right]}_{\text {see also Eq. }(6)}=\sum_{\xi=1}^{q} 1.0=q \\
\left(\overline{\mathbf{A}}^{\mathbf{T}} \cdot \overline{\mathbf{B}}\right)_{1 \times 1} & =\sum_{\xi=1}^{q}\left[\gamma_{x}(\xi) \cdot A_{\text {bal }}(\xi)+\gamma_{y}(\xi) \cdot S_{\text {bal }}(\xi)+\gamma_{z}(\xi) \cdot N_{\text {bal }}(\xi)\right]
\end{aligned}
$$

Then, after using the right-hand sides of Eq. (23a) and (23b) in Eq. (22) and after recognizing that the "inverse" of the "matrix" used on the left-hand side of Eq. (23a) equals $1 / q$, we get:

$$
\widehat{w}=\frac{1}{q} \cdot \sum_{\xi=1}^{q}\left[\gamma_{x}(\xi) \cdot A_{b a l}(\xi)+\gamma_{y}(\xi) \cdot S_{b a l}(\xi)+\gamma_{z}(\xi) \cdot N_{b a l}(\xi)\right]
$$

It is possible to simplify Eq. (24) even further. The summation term on the right-hand side of Eq. (24) can be written as a scalar product of two vectors. Then, we get:

$$
\boldsymbol{\Gamma}(\xi) \cdot \mathbf{W}(\xi)=\gamma_{x}(\xi) \cdot A_{b a l}(\xi)+\gamma_{y}(\xi) \cdot S_{b a l}(\xi)+\gamma_{z}(\xi) \cdot N_{b a l}(\xi)
$$


In general, the scalar product of two vectors can also be expressed as the product of their lengths and the cosine of the angle between them. Therefore, we can write:

$$
\boldsymbol{\Gamma}(\xi) \cdot \mathbf{W}(\xi)=|\boldsymbol{\Gamma}(\xi)| \cdot|\mathbf{W}(\xi)| \cdot \cos \eta(\xi)
$$

The length of the two vectors used in Eq. (26) is given by the following equations:

$$
\begin{gathered}
|\boldsymbol{\Gamma}(\xi)|=1.0 \\
|\mathbf{W}(\xi)|=\sqrt{A_{b a l}^{2}(\xi)+S_{b a l}^{2}(\xi)+N_{b a l}^{2}(\xi)}
\end{gathered}
$$

In addition, we conclude from Eq. (7) that the two vectors are parallel to each other. Therefore, we get:

$$
E q .(7) \quad \Longrightarrow \quad \Gamma(\xi) \| \mathbf{W}(\xi) \quad \Longrightarrow \quad \eta(\xi)=0 \quad \Longrightarrow \quad \cos \eta(\xi)=1
$$

Now, after using the right-hand sides of Eqs. (27a), (27b) and (27c) on the right-hand side of Eq. (26), we get for the scalar product of the two vectors the expression:

$$
\boldsymbol{\Gamma}(\xi) \cdot \mathbf{W}(\xi)=\sqrt{A_{b a l}^{2}(\xi)+S_{b a l}^{2}(\xi)+N_{b a l}^{2}(\xi)}
$$

Then, after comparing Eq. (28) with Eq. (25), we get the following identity:

$$
\gamma_{x}(\xi) \cdot A_{b a l}(\xi)+\gamma_{y}(\xi) \cdot S_{b a l}(\xi)+\gamma_{z}(\xi) \cdot N_{b a l}(\xi)=\sqrt{A_{b a l}^{2}(\xi)+S_{b a l}^{2}(\xi)+N_{b a l}^{2}(\xi)}
$$

Finally, after substituting the summation term of Eq. (24) by the right-hand side of Eq. (29), we get the following explicit solution for the least squares estimator of the model weight (the corresponding confidence interval on the model weight is derived in App. 1):

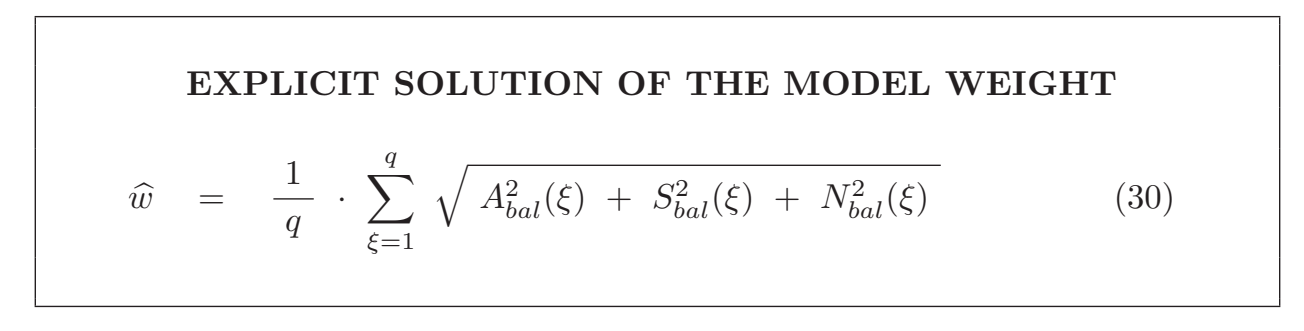

A useful interpretation of Eq. (30) can be made $\Longrightarrow$ the least squares estimator of the model weight equals the arithmetic mean of the individual weights that are calculated for each "wind-off" data point. It is also concluded from Eq. (30) that the accuracy of the model weight prediction is directly linked to the accuracy of the axial, side, and normal force predictions of the balance. The accuracies of these force predictions, assuming they are described in engineering units, differ significantly for a typical six-component strain-gage balance because the axial force capacity is often one order of magnitude smaller than the normal force capacity. In other words - the prediction error of the axial force is expected to be substantially smaller than the corresponding error of the normal force. This conclusion suggests that the wind tunnel model should be pitched up as high as possible in order to maximize the contribution of the axial force in Eq. (30). Unfortunately, the pitch angle range of a typical wind tunnel model goes from $\mu \approx-5^{\circ}$ to $\mu \approx+20^{\circ}$. Consequently, the error associated with the normal force prediction will dominate the total error associated with the model weight prediction for the majority of wind tunnel models (an upper bound of the model weight prediction error is derived in App. 2; a more general error analysis is described in App. 5).

\section{Least Squares Estimators of the Center of Gravity Coordinates}

Now, the remaining least squares estimators of the center of gravity coordinates of the wind tunnel model have to be determined. The new method uses a second least squares fit of the "wind-off" data points for this purpose. The second least squares problem is defined by assuming that the weight of the model is 
known as the result of the first least squares fit. This weight, i.e., the least squares estimator $\widehat{w}$, is defined in Eq. (30). In addition, the second least squares fit is constructed by exclusively using the predicted moments of the "wind-off" data points. Then, after applying Eqs. (14a) to (14c), we get the following equations for a single "wind-off" data point with index $\xi$ :

$$
\begin{aligned}
& R_{\text {bal }}(\xi)=\gamma_{z}(\xi) \cdot \widehat{w} \cdot \underbrace{d_{y}}_{\text {unknown }}-\gamma_{y}(\xi) \cdot \widehat{w} \cdot \underbrace{d_{z}}_{\text {unknown }} ; 1 \leq \xi \leq q \\
& P_{b a l}(\xi)=\gamma_{x}(\xi) \cdot \widehat{w} \cdot \underbrace{d_{z}}_{\text {unknown }}-\gamma_{z}(\xi) \cdot \widehat{w} \cdot \underbrace{d_{x}}_{\text {unknown }} ; 1 \leq \xi \leq q \\
& Y_{\text {bal }}(\xi)=\gamma_{y}(\xi) \cdot \widehat{w} \cdot \underbrace{d_{x}}_{\text {unknown }}-\gamma_{x}(\xi) \cdot \widehat{w} \cdot \underbrace{d_{y}}_{y} ; 1 \leq \xi \leq q
\end{aligned}
$$

Equations $(31 a)$ to $(31 c)$ can be expressed in matrix format. Then, we get:

$$
\underbrace{\left[\begin{array}{ccc}
0 & +\gamma_{z}(\xi) \cdot \widehat{w} & -\gamma_{y}(\xi) \cdot \widehat{w} \\
-\gamma_{z}(\xi) \cdot \widehat{w} & 0 & +\gamma_{x}(\xi) \cdot \widehat{w} \\
+\gamma_{y}(\xi) \cdot \widehat{w} & -\gamma_{x}(\xi) \cdot \widehat{w} & 0
\end{array}\right]}_{\text {model attitude measurement and weight from Eq. }(30)} \cdot \underbrace{\left[\begin{array}{c}
d_{x} \\
d_{y} \\
d_{z}
\end{array}\right]}_{\text {unknown }}=\underbrace{\left[\begin{array}{c}
R_{\text {bal }}(\xi) \\
P_{\text {bal }}(\xi) \\
Y_{\text {bal }}(\xi)
\end{array}\right]}_{\text {balance load }}
$$

In the next step, abbreviations of the matrix and vectors used in Eq. (32) are introduced. In addition, the coordinates $d_{x}, d_{y}$, and $d_{z}$ are replaced by their least squares estimators $\widehat{d}_{x}, \widehat{d}_{y}$, and $\widehat{d}_{z}$. Then, we get:

$$
\begin{aligned}
& \widehat{\mathbf{K}}(\xi)_{3 \times 3}=\left[\begin{array}{ccc}
0 & +\gamma_{z}(\xi) \cdot \widehat{w} & -\gamma_{y}(\xi) \cdot \widehat{w} \\
-\gamma_{z}(\xi) \cdot \widehat{w} & 0 & +\gamma_{x}(\xi) \cdot \widehat{w} \\
+\gamma_{y}(\xi) \cdot \widehat{w} & -\gamma_{x}(\xi) \cdot \widehat{w} & 0
\end{array}\right] \\
& \widehat{\mathbf{L}}(\xi)_{3 \times 1}=\left[\begin{array}{c}
R_{b a l}(\xi) \\
P_{b a l}(\xi) \\
Y_{b a l}(\xi)
\end{array}\right] \\
& \widehat{\mathbf{X}}_{3 \times 1}=\left[\begin{array}{c}
\widehat{d}_{x} \\
\widehat{d}_{y} \\
\widehat{d}_{z}
\end{array}\right]
\end{aligned}
$$

Consequently, Eq. (32) can be expressed as follows for a single "wind-off" data point with index $\xi$ :

$$
\widehat{\mathbf{K}}(\xi)_{3 \times 3} \cdot \widehat{\mathbf{X}}_{3 \times 1}=\widehat{\mathbf{L}}(\xi)_{3 \times 1}
$$

In addition, we get the following equation if Eq. (34a) is expanded to all "wind-off" data points:

$$
\left[\begin{array}{c}
\widehat{\mathbf{K}}(1)_{3 \times 3} \\
\widehat{\mathbf{K}}(2)_{3 \times 3} \\
\cdots \\
\widehat{\mathbf{K}}(q)_{3 \times 3}
\end{array}\right] \cdot \widehat{\mathbf{X}}_{3 \times 1}=\left[\begin{array}{c}
\widehat{\mathbf{L}}(1)_{3 \times 1} \\
\widehat{\mathbf{L}}(2)_{3 \times 1} \\
\cdots \\
\widehat{\mathbf{L}}(q)_{3 \times 1}
\end{array}\right]
$$

For convenience, the matrix and vector associated with the data points can be abbreviated as follows:

$$
\widehat{\mathbf{A}}_{3 q \times 3}=\left[\begin{array}{c}
\widehat{\mathbf{K}}(1)_{3 \times 3} \\
\widehat{\mathbf{K}}(2)_{3 \times 3} \\
\cdots \\
\widehat{\mathbf{K}}(q)_{3 \times 3}
\end{array}\right]
$$




$$
\widehat{\mathbf{B}}_{3 q \times 1}=\left[\begin{array}{c}
\widehat{\mathbf{L}}(1)_{3 \times 1} \\
\widehat{\mathbf{L}}(2)_{3 \times 1} \\
\cdots \\
\widehat{\mathbf{L}}(q)_{3 \times 1}
\end{array}\right]
$$

Then, after using Eqs. (35a) and (35b) to simplify Eq. (34b), the equations of the second global regression analysis problem are obtained. We get:

$$
\text { Second Global Regression Analysis Problem } \Longrightarrow \widehat{\mathbf{A}}_{3 q \times 3} \cdot \widehat{\mathbf{X}}_{3 \times 1}=\widehat{\mathbf{B}}_{3 q \times 1}
$$

The solution of the second global regression problem can be described by the following matrix equations:

\section{LEAST SQUARES ESTIMATORS OF THE CENTER OF GRAVITY COORDINATES}

$$
\begin{gathered}
\left(\widehat{\mathbf{A}}^{\mathbf{T}} \widehat{\mathbf{A}}\right)_{3 \times 3} \cdot \widehat{\mathbf{X}}_{3 \times 1}=\left(\widehat{\mathbf{A}}^{\mathbf{T}} \widehat{\mathbf{B}}\right)_{3 \times 1} \\
\widehat{\mathbf{X}}_{3 \times 1}=\left[\begin{array}{c}
\widehat{d}_{x} \\
\widehat{d}_{y} \\
\widehat{d}_{z}
\end{array}\right]=\left(\widehat{\mathbf{A}}^{\mathbf{T}} \widehat{\mathbf{A}}\right)_{3 \times 3}^{-1} \cdot\left(\widehat{\mathbf{A}}^{\mathbf{T}} \widehat{\mathbf{B}}\right)_{3 \times 1}
\end{gathered}
$$

The solution of the second global regression problem is expressed in two different ways. The first matrix equation, i.e., Eq. (37a), represents the "normal equations" of the global regression problem. They define the solution as a $3 \times 3$ linear system of equations that has to be solved for the three unknown center of gravity coordinates contained in vector $\widehat{\mathbf{X}}$. The second matrix equation, i.e., Eq. (37b), is an "academic" solution of the linear system that is defined in Eq. (37a). It expresses the solution as a matrix multiplication assuming that the inverse matrix used on the right-hand side of Eq. (37b) has been determined.

An explicit solution of Eq. (37a) is derived in App. 3. In addition, the confidence intervals on the center of gravity coordinates are derived in App. 4. It is always helpful if the center of gravity coordinate predictions can independently be verified. The center of gravity of a model with symmetric weight distribution, for example, has to be located in the plane that is defined by the axial force and the normal force in the balance axis system. Therefore, the new method's predicted least squares estimator $\widehat{d}_{y}$ of the center of gravity coordinate $d_{y}$ has to be close to zero for a model with a symmetric weight distribution.

\section{Selection of "Wind-Off" Data Points}

The "wind-off" data points for the global regression analysis of the weight and center of gravity coordinates of the wind tunnel model have to be selected carefully so that (i) the confidence intervals on the corresponding least squares estimators are meaningful and that (ii) the linear system associated with the second global regression analysis problem, i.e., Eq. (37a), has a unique solution. It is shown in the literature that a unique solution of a general global regression analysis problem exists if the columns of the matrix of the regressors, i.e., matrix $\widehat{\mathbf{A}}$ of Eq. (35a), are linearly independent (for more detail see Ref. [4], p. 69).

Different approaches are used in the aerospace testing community to select "wind-off" data points for the determination of the model's weight and center of gravity coordinates. The authors, for example, recommend to use at least five "evenly" distributed data points for the global regression analysis in order to have sufficiently small confidence intervals on the least squares estimators (see App. 1 and App. 4 for a discussion of the connection between the size of the confidence interval on the least squares estimators and the number of "wind-off" data points). The bounds of the data point set could be defined by the model's largest positive pitch angle $\left(\mu_{\max }\right)$ and the model's largest negative pitch angle $\left(\mu_{\min }\right)$. In addition, the "wind-off" data point at "zero" pitch angle $\left(\mu=0^{\circ}\right)$ could be used as a "confirmation point" in order to independently validate the prediction of the model weight and center of gravity coordinates that are the result of the least 
squares fits of the five "wind-off" data points. Table 1 below lists this first set of recommended "wind-off" data points including the confirmation point:

Table 1: First set of "wind-off" data points $\left(\gamma_{y}=0 ; S_{b a l}=0 ; q=5\right.$; one confirmation point).

\begin{tabular}{|c|c|c|c|c|}
\hline$\xi$ & Pitch Angle & Roll Angle & $A_{b a l}$ & $N_{b a l}$ \\
\hline 1 & $\mu(1)=\mu_{\min }<0^{\circ}$ & $0^{\circ}$ & $A_{b a l}(1)$ & $N_{b a l}(1)$ \\
\hline 2 & $\mu(2)=\mu_{\min }+0.25 \cdot\left(\mu_{\max }-\mu_{\min }\right)$ & $0^{\circ}$ & $A_{b a l}(2)$ & $N_{b a l}(2)$ \\
\hline 3 & $\mu(3)=\mu_{\min }+0.50 \cdot\left(\mu_{\max }-\mu_{\min }\right)$ & $0^{\circ}$ & $A_{b a l}(3)$ & $N_{b a l}(3)$ \\
\hline 4 & $\mu(4)=\mu_{\min }+0.75 \cdot\left(\mu_{\max }-\mu_{\min }\right)$ & $0^{\circ}$ & $A_{b a l}(4)$ & $N_{b a l}(4)$ \\
\hline 5 & $\mu(5)=\mu_{\max }>0^{\circ}$ & $0^{\circ}$ & $A_{b a l}(5)$ & $N_{b a l}(5)$ \\
\hline 6 & $\mu(6)=0^{\circ}$ (Conf. Pt.) & $0^{\circ}$ & $\left|A_{b a l}\right| \approx 0$ & $\left|N_{b a l}\right| \approx w$ \\
\hline
\end{tabular}

It was mentioned during the discussion of the explicit solution of the model weight, i.e., Eq. (30), that the accuracy of the normal force prediction has a substantial influence on the total error associated with the model weight calculation (see also the error analysis that is discussed in App. 2). Therefore, whenever possible, the authors recommend to record "wind-off" data points with the model in both "upright" and "inverted" orientation, i.e., for the model roll angles $0^{\circ}$ and $180^{\circ}$. In this case, the normal force will have both positive and negative signs which may help reduce a potential "hidden" bias in the experimental data that may exist if the normal force of the "wind-off" data points never changes sign. Table 2 below shows, for example, "wind-off" data points for a model in both "upright" and "inverted" orientation (the two "wind-off" confirmation points at "zero" pitch angle, i.e., $\mu=0^{\circ}$, are also listed).

Table 2: Second set of "wind-off" data points $\left(\gamma_{y}=0 ; S_{b a l}=0 ; q=10\right.$; two confirmation points).

\begin{tabular}{|c|c|c|c|c|}
\hline$\xi$ & Pitch Angle & Roll Angle & $A_{\text {bal }}$ & $N_{\text {bal }}$ \\
\hline \hline 1 & $\mu(1)$ & $0^{\circ}$ & $A_{b a l}(1)$ & $N_{\text {bal }}(1)$ \\
\hline 2 & $\mu(2)$ & $0^{\circ}$ & $A_{b a l}(2)$ & $N_{b a l}(2)$ \\
\hline 3 & $\mu(3)$ & $0^{\circ}$ & $A_{b a l}(3)$ & $N_{b a l}(3)$ \\
\hline 4 & $\mu(4)$ & $0^{\circ}$ & $A_{b a l}(4)$ & $N_{b a l}(4)$ \\
\hline 5 & $\mu(5)$ & $0^{\circ}$ & $A_{b a l}(5)$ & $N_{b a l}(5)$ \\
\hline 6 & $\mu(6)=\mu(1)$ & $180^{\circ}$ & $A_{b a l}(6) \approx-A_{b a l}(1)$ & $N_{b a l}(6) \approx-N_{b a l}(1)$ \\
\hline 7 & $\mu(7)=\mu(2)$ & $180^{\circ}$ & $A_{b a l}(7) \approx-A_{b a l}(2)$ & $N_{b a l}(7) \approx-N_{b a l}(2)$ \\
\hline 8 & $\mu(8)=\mu(3)$ & $180^{\circ}$ & $A_{b a l}(8) \approx-A_{b a l}(3)$ & $N_{b a l}(8) \approx-N_{b a l}(3)$ \\
\hline 9 & $\mu(9)=\mu(4)$ & $180^{\circ}$ & $A_{b a l}(9) \approx-A_{b a l}(4)$ & $N_{b a l}(9) \approx-N_{b a l}(4)$ \\
\hline 10 & $\mu(10)=\mu(5)$ & $180^{\circ}$ & $A_{b a l}(10) \approx-A_{b a l}(5)$ & $N_{b a l}(10) \approx-N_{b a l}(5)$ \\
\hline \hline 11 & $\mu(11)=0^{\circ}($ Conf. Pt. 1$)$ & $0^{\circ}$ & $\left|A_{b a l}\right| \approx 0$ & $\left|N_{b a l}\right| \approx w$ \\
\hline 12 & $\mu(12)=0^{\circ}($ Conf. Pt. 2$)$ & $180^{\circ}$ & $\left|A_{b a l}\right| \approx 0$ & $\left|N_{b a l}\right| \approx w$ \\
\hline
\end{tabular}

The selection of "wind-off" data point suggested in both Table 1 and Table 2 may potentially be improved by choosing data points such that the variance in the regression coefficients, i.e, the variance in the model weight and in the center of gravity coordinates, is minimized. This goal can be achieved by using a point selection that maximizes the determinantes of $\overline{\mathbf{A}}^{\mathbf{T}} \overline{\mathbf{A}}$ and $\widehat{\mathbf{A}}^{\mathbf{T}} \widehat{\mathbf{A}}$ (a discussion of the related experimental design problem can be found in Ref. [4], p. 505/506).

Unfortunately, the aerospace testing community traditionally uses two load sign definitions that do 
not follow the signs of the related Cartesian coordinates of a right-handed coordinate system (see, e.g., the balance axis system definition used in Ref. [1], p. 3). Therefore, load transformations may have to be performed in order to apply the new method to traditional wind tunnel test data. These transformations are discussed in the next section of the paper.

\section{Balance Load Transformations}

The "primary" balance axis system is shown in Fig. 2. It is a right-handed coordinate system. It also uses sign definitions for both the forces and moments that match sign definitions of related Cartesian coordinates. Only in this case the cross-product operator of vector algebra can be used for the calculation of moments that are associated with model weight effects. Therefore, the "primary" balance axis system was chosen for the rigorous derivations of all model weight corrections that are discussed in this paper.

The aerospace testing community, on the other hand, uses an "alternate" balance axis system. This "alternate" balance axis system is depicted in Fig. 5. Its coordinate and load sign definitions were directly copied from the literature (taken from Ref. [1], p. 3, Fig. 1). Unfortunately, the "alternate" balance axis system has two load components that contradict the sign definitions of related Cartesian coordinates. Therefore, the "alternate" balance axis system has the disadvantage that the cross-product operator of vector algebra cannot be used for the calculation of moments that are associated with model weight effects. Consequently, the "alternate" balance axis system is not suited for a rigorous "first-principles-based" derivation of the model weight corrections.

Differences between the "primary" and "alternate" balance axis systems and associated load sign definitions can most easily be recognized by viewing Fig. 2 and Fig. 5 side-by-side. It can be seen that the coordinate axes and load sign definitions of Fig. 2 and Fig. 5 are identical with the exception of the sign definitions of the axial and normal forces. The positive axial force of the "primary" balance axis system points in the direction of the positive $x$-axis. The positive axial force of the "alternate" balance axis system points in the direction of the negative $x$-axis. The positive normal force of the "primary" balance axis system points in the direction of the positive $z$-axis. The positive normal force of the "alternate" balance axis system points in the direction of the negative $z$-axis.

Fortunately, it is possible to use all results presented in this paper for the "primary" axis system with the traditional load sign definition of the aerospace testing community. It is only required to perform two load transformations. First, assuming that balance loads follow the traditional sign convention used in the aerospace testing community (see Fig. 5), the axial force and normal force of the "wind-off" data points are transformed from the "alternate" to the "primary" balance axis system by using the following relationships:

$$
\begin{aligned}
& A_{b a l}=(-1) \cdot A F \\
& N_{b a l}=(-1) \cdot N F
\end{aligned}
$$

All other balance load components defined in the "alternate" balance axis system can directly be used with the results presented in this paper. Therefore, we get:

$$
\begin{aligned}
S_{b a l} & =S F \\
R_{b a l} & =R M \\
P_{b a l} & =P M \\
Y_{b a l} & =Y M
\end{aligned}
$$

Then, the model weight corrections of the six balance loads are determined as outlined in the previous sections. Finally, the model weight corrections of the axial and normal force have to be transformed back from the "primary" to the "alternate" balance axis system. The following transformations apply:

$$
\begin{aligned}
& A F_{\text {weight }}=(-1) \cdot A_{\text {weight }} \\
& N F_{\text {weight }}=(-1) \cdot N_{\text {weight }}
\end{aligned}
$$


All other model weight corrections, i.e., $S_{\text {weight }}, R_{\text {weight }}, P_{\text {weight }}$, and $Y_{\text {weight }}$ can directly be applied to the original balance loads as no sign change is required. The following relationships apply:

$$
\begin{aligned}
S F_{\text {weight }} & =S_{\text {weight }} \\
R M_{\text {weight }} & =R_{\text {weight }} \\
P M_{\text {weight }} & =P_{\text {weight }} \\
Y M_{\text {weight }} & =Y_{\text {weight }}
\end{aligned}
$$

It is important to point out that the Cartesian coordinate definitions of the "primary" and "alternate" balance axis systems are identical. Therefore, no sign transformations need to be applied to the least squares estimators of the center of gravity coordinates. They are valid in both balance axis systems.

\section{Conclusions}

A new method for the prediction of wind tunnel model weight corrections for strain-gage balance loads was introduced. The new method became possible after rigorously applying the ideas of global regression analysis to a set of "wind-off" data points that are needed for the prediction of the least squares estimators of the weight and center of gravity coordinates of the wind tunnel model. Figure 6 summarizes basic elements of the new method to predict model weight corrections.

For the first time, a complete set of explicit equations is published that may be used for the determination of the least squares estimators of the model weight and center of gravity coordinates. In addition, explicit equations of the confidence intervals on the model weight and center of gravity coordinates are listed in the appendices of the paper. This complete set of explicit equations greatly simplifies the use of the new method in the data system of a wind tunnel facility as complexities associated with the implementation of a global regression analysis algorithm are avoided.

The new method uses two separate least squares fits for the determination of the model weight and center of gravity coordinates. First, it is proven that the least squares estimator of the model weight equals the arithmetic mean of the model weights of the individual "wind-off" data points that are used for the global regression analysis. Then, least squares estimators of the center of gravity coordinates of the model are derived as the explicit solution of a $3 \times 3$ linear system of equations. In addition, two different error analyses of the model weight are discussed in the appendices of the paper. Finally, recommendations for suitable sets of "wind-off" data points are made that may be used for the determination of wind tunnel model weight corrections.

\section{Acknowledgements}

The authors want to thank Richard DeLoach of NASA Langley Research Center for his critical and constructive review of the final manuscript. He contributed parts of the general error analysis of the model weight prediction that is discussed in App. 5 of the paper. The authors are also grateful to the late Alan Boone of NASA Ames Research Center who first suggested to rigorously use global regression analysis for the prediction of the weight and center of gravity coordinates of a wind tunnel model. The work reported in this paper was partially supported by the Wind Tunnel Division at NASA Ames Research Center under contract NNA09DB39C.

\section{References}

${ }^{1}$ American Institute of Aeronautics and Astronautics, Recommended Practice - Calibration and Use of Internal Strain Gage Balances with Application to Wind Tunnel Testing, AIAA R-091-2003, American Institute of Aeronautics and Astronautics, Reston, Virginia, 2003; p. 3, pp. 18-19.

${ }^{2}$ Ulbrich, N. and Volden, T., "Strain-Gage Balance Calibration Analysis using Automatically Selected Math Models," AIAA 2005-4084, paper presented at the 41st AIAA/ASME/SAE/ASEE Joint Propulsion Conference and Exhibit, Tucson, Arizona, July 2005; Eq. (25), Eqs. (32a) to (32c). 
${ }^{3}$ Fishbane, P. M., Gasiorowicz, S., Thornton, S. T., Physics for Scientists and Engineers, extended version, Prentice-Hall, Inc., Englewood Cliffs, New Jersey, 1993; p. 127, p. 302.

${ }^{4}$ Montgomery, D. C., Peck, E. A., and Vining, G. G., Introduction to Linear Regression Analysis, 4th ed., John Wiley \& Sons, Inc., New York, 2006; pp. 41-42, pp. 63-77, p. 81, p. 93/94, p. 505/506, p. 515.

${ }^{5}$ Burden, R. L. and Faires, J. D., Numerical Analysis, 3rd edition, PWS-Kent Publishing Company, Boston, Massachusetts, 1985; pp. 323-327 (Algorithm 6.2), p. 409, Eq. (8.1).

${ }^{6}$ Strang, G., Introduction to Applied Mathematics, Wellesley-Cambridge Press, Wellesley, Massachusetts, 1986; pp. 35-39.

${ }^{7}$ Press, W. H., Teukolsky, S. A., Vetterling, W. T., Flannery, B. P., Numerical Recipes in Fortran 77, 2nd edition, reprinted with corrections, Cambridge University Press, Cambridge, New York, 1996; pp. 665-675.

${ }^{8}$ Coleman, H. W., and Steele, W. G., Experimentation and Uncertainty Analysis for Engineers, 2nd edition, John Wiley \& Sons, Inc., Wiley-Interscience Publication, New York, 1999, p. 49.

\section{List of Appendices}

\begin{tabular}{|c|c|c|c|}
\hline No. & Appendix Title & Page Range & Contents Summary \\
\hline 1 & Confidence Interval on the Model Weight & pp. $17-20$ & $\begin{array}{l}\ldots \text { describes a rigorous } \\
\text { derivation of the confidence } \\
\text { interval on the model weight. }\end{array}$ \\
\hline 2 & Upper Bound of the Model Weight Prediction Error & pp. $21-23$ & $\begin{array}{l}\text {... describes the derivation of } \\
\text { a Taylor Series approximation } \\
\text { of an upper bound of the } \\
\text { model weight prediction error. }\end{array}$ \\
\hline 3 & Explicit Solution of the Center of Gravity Coordinates & pp. $24-25$ & $\begin{array}{l}\text {.. describes an explicit } \\
\text { solution of the center of } \\
\text { gravity coordinates that is } \\
\text { based on Cramer's Rule. }\end{array}$ \\
\hline 4 & Confidence Intervals on the Center of Gravity Coordinates & pp. $26-30$ & $\begin{array}{l}\ldots \text { uses Reference [4] to } \\
\text { get explicit solutions of the } \\
\text { confidence intervals on the } \\
\text { center of gravity coordinates. }\end{array}$ \\
\hline 5 & General Error Analysis of the Model Weight Prediction & pp. 31-33 & $\begin{array}{l}\text {... describes a derivation of } \\
\text { the variance in the model } \\
\text { weight prediction and a } \\
\text { theoretical estimate of the } \\
\text { required minimum number } \\
\text { of "wind-off" data points. }\end{array}$ \\
\hline
\end{tabular}




\section{Appendix 1: Confidence Interval on the Model Weight}

The wind tunnel model weight was introduced as a regression coefficient in an earlier section of the paper. The model weight was obtained after fitting strain-gage balance forces as a function of related model attitude measurements. The reporting of results of such a regression analysis becomes more complete if confidence intervals on the regression coefficients are estimated. Therefore, explicit equations for the confidence interval on the model weight are discussed in this section. These equations were derived from more general relationships that are reported in the literature (see Ref. [4], pp. 41-42). This approach was selected as a derivation of the general equations of the confidence intervals goes beyond the scope of the present paper. First, the general equations of the confidence interval on a regression coefficient of a "nointercept" model are developed using information that is provided in the literature. Then, it is shown how these general equations can be applied to the wind tunnel model weight.

The discussion of the general equations of the confidence interval on a regression coefficient starts with a "no-intercept" regression model as this type of model was used to obtain the model weight (see Eqs. (17), $(19 b))$. This "no-intercept" model can be described as follows (see Ref. [4], Eq. (2.48)):

$$
y=\beta_{1} \cdot x
$$

where $x$ is the regressor variable, $y$ is the response variable, and $\beta_{1}$ is the regression coefficient. Furthermore, it is assumed that a total of $n$ observations, i.e., $\left(y_{i}, x_{i}\right), i=1,2, \ldots, n$, are to be used to determine the least squares estimator $\widehat{\beta}_{1}$ of the slope $\beta_{1}$. Then, Eq. (40) can be expressed for each observation by using the following equation:

$$
y_{i}=\beta_{1} \cdot x_{i} ; 1 \leq i \leq n
$$

Similarities between the general "no-intercept" model discussed in Ref. [4], pp. 41-42, and the model used for the determination of the model weight exist that can be recognized after comparing terms of Eq. (19b) with terms of Eq. (41). Table 3 below summarizes these similarities:

Table 3: Comparison of the Nomenclature used for the Model Weight Determination.

\begin{tabular}{|c|c|c|}
\hline Reference [4] & present paper & Comments \\
\hline \hline$\beta_{1}$ & $w$ & regression coefficient; model weight \\
\hline$\widehat{\beta}_{1}$ & $\widehat{w}$ & least squares estimator; Eq. (30) \\
\hline$n$ & $3 q$ & total number of "observations" \\
\hline$x_{1}, x_{2}, \ldots, x_{n}$ & $\gamma_{x}(1), \gamma_{y}(1), \gamma_{z}(1), \ldots, \gamma_{y}(q), \gamma_{z}(q)$ & regressor variable values \\
\hline$y_{1}, y_{2}, \ldots, y_{n}$ & $A_{b a l}(1), S_{b a l}(1), N_{b a l}(1), \ldots, S_{b a l}(q), N_{b a l}(q)$ & response variable values \\
\hline
\end{tabular}

The analytic solution of the regression coefficient of the "no-intercept" regression model is given in the literature as follows (from Ref. [4], Eq. (2.50)):

$$
\widehat{\beta}_{1}=\frac{\sum_{i=1}^{n} y_{i} x_{i}}{\sum_{i=1}^{n} x_{i}^{2}}
$$

Now, the question comes up if the regression solution of the model weight (i.e., Eq. (24) and Eq. (30)) can be obtained "by inspection" from the general solution of the regression coefficient of the "no-intercept" model that is given in Ref. [4] (see also Eq. (42) above). In that case, the confidence interval on the model weight could directly be deduced from the confidence interval on the regression coefficient of the general "no-intercept" model that is listed in Ref. [4]. The answer to the question can be found by using the nomenclature comparison shown in Table 3 for the replacement of $\widehat{\beta}_{1}, x_{i}$, and $y_{i}$ in Eq. (42). Then, we get:

$$
\widehat{w}=\frac{A_{b a l}(1) \cdot \gamma_{x}(1)+S_{b a l}(1) \cdot \gamma_{y}(1)+\cdots+S_{b a l}(q) \cdot \gamma_{y}(q)+N_{b a l}(q) \cdot \gamma_{z}(q)}{\gamma_{x}^{2}(1)+\gamma_{y}^{2}(1)+\cdots+\gamma_{y}^{2}(q)+\gamma_{z}^{2}(q)}
$$


Equation (43) can also be expressed as follows:

$$
\widehat{w}=\frac{\sum_{\xi=1}^{q}\left[A_{b a l}(\xi) \cdot \gamma_{x}(\xi)+S_{b a l}(\xi) \cdot \gamma_{y}(\xi)+N_{b a l}(\xi) \cdot \gamma_{z}(\xi)\right]}{\sum_{\xi=1}^{q}\left[\gamma_{x}^{2}(\xi)+\gamma_{y}^{2}(\xi)+\gamma_{z}^{2}(\xi)\right]}
$$

We also know from Eq. (23a) that the following relationship applies:

$$
q=\sum_{\xi=1}^{q}\left[\gamma_{x}^{2}(\xi)+\gamma_{y}^{2}(\xi)+\gamma_{z}^{2}(\xi)\right]
$$

Then, after replacing the denominator of Eq. (44) with the left hand side of Eq. (45) and after rearranging the factors of the products of the summation terms of the numerator of Eq. (44), we get:

$$
\widehat{w}=\frac{1}{q} \cdot \sum_{\xi=1}^{q}\left[\gamma_{x}(\xi) \cdot A_{b a l}(\xi)+\gamma_{y}(\xi) \cdot S_{b a l}(\xi)+\gamma_{z}(\xi) \cdot N_{b a l}(\xi)\right]
$$

As expected, the right hand side of Eq. (46) exactly matches the right hand side of Eq. (24). Therefore, the general least squares solution of the regression coefficient of the "no-intercept" model from the literature (i.e., Eq. (42) above, or, Ref. [4], Eq. (2.50)) matches the solution for the model weight that was developed in an earlier section of the current paper (i.e., Eq. (24) and Eq. (30)). Consequently, it is possible to use the formula of the confidence interval from the literature (Ref. [4], Eq. (2.53)) to directly develop the confidence interval on the model weight. It is only necessary to replace the generic nomenclature used for the definition of the confidence interval in Ref. [4] with the nomenclature that is associated with the determination of the model weight.

Reference [4] constructs the confidence interval on the regression coefficient $\beta_{1}$ by making the normality assumption on the errors of the least squares fit. Then, the $100(1-\alpha)$ percent confidence interval on the slope $\beta_{1}$ of the "no-intercept" model is defined as follows (from Ref. [4], Eqs. (2.52), (2.53)):

\section{CONFIDENCE INTERVAL ON THE SLOPE OF A "NO-INTERCEPT" MODEL} (... taken from Ref. [4], Eqs. $(2.52),(2.53), \ldots)$

$$
\widehat{\beta}_{1}-t_{\alpha / 2, n-1} \sqrt{\frac{M S_{\text {Res }}}{\sum_{i=1}^{n} x_{i}^{2}}} \leq \beta_{1} \leq \widehat{\beta}_{1}+t_{\alpha / 2, n-1} \sqrt{\frac{M S_{\text {Res }}}{\sum_{i=1}^{n} x_{i}^{2}}}
$$

where the residual mean square $M S_{\text {Res }}$ is defined as

$$
M S_{\text {Res }}=\frac{1}{n-1} \cdot\left[\sum_{i=1}^{n} y_{i}^{2}-\widehat{\beta}_{1} \sum_{i=1}^{n} y_{i} x_{i}\right]
$$

Equations (47a) and (47b) can be used to construct the confidence interval on the model weight. It is only required to replace the generic terms by the corresponding wind tunnel model specific terms. For example, the following two relationships can be obtained from Table 3 and Eq. (46) above:

$$
n=3 q
$$




$$
\widehat{\beta}_{1}=\widehat{w}=\frac{1}{q} \cdot \sum_{\xi=1}^{q}\left[\gamma_{x}(\xi) \cdot A_{b a l}(\xi)+\gamma_{y}(\xi) \cdot S_{b a l}(\xi)+\gamma_{z}(\xi) \cdot N_{b a l}(\xi)\right]
$$

It was shown above that the right hand side of Eq. (24) equals the right hand side of Eq. (46) (i.e., Eq. (49)). In addition, it was proven earlier that the right hand side of Eq. (24) equals the right hand side of Eq. (30). Therefore, after replacing the right hand side of Eq. (49) with the right hand side of Eq. (30), we get the following simplified relationship:

$$
\widehat{\beta}_{1}=\widehat{w}=\frac{1}{q} \cdot \sum_{\xi=1}^{q} \sqrt{A_{b a l}^{2}(\xi)+S_{b a l}^{2}(\xi)+N_{b a l}^{2}(\xi)}
$$

Now, after using the contents of Table 3 to replace $x_{i}$ and after applying Eq. (23a) to the result, we get:

$$
\sum_{i=1}^{n} x_{i}^{2}=\gamma_{x}^{2}(1)+\gamma_{y}^{2}(1)+\cdots+\gamma_{z}^{2}(q)=\sum_{\xi=1}^{q}\left[\gamma_{x}^{2}(\xi)+\gamma_{y}^{2}(\xi)+\gamma_{z}^{2}(\xi)\right]=q
$$

Similarly, after using the contents of Table 3 to replace $y_{i}$, we get:

$$
\sum_{i=1}^{n} y_{i}^{2}=A_{b a l}^{2}(1)+S_{b a l}^{2}(1)+\cdots+N_{b a l}^{2}(q)=\sum_{\xi=1}^{q}\left[A_{b a l}^{2}(\xi)+S_{b a l}^{2}(\xi)+N_{b a l}^{2}(\xi)\right]
$$

We also know, after rearranging Eq. (42), that the following relationship applies:

$$
\sum_{i=1}^{n} y_{i} x_{i}=\widehat{\beta}_{1} \cdot \sum_{i=1}^{n} x_{i}^{2}
$$

The right hand side of Eq. (53) can be expressed differently. First, the right hand side of Eq. (50) can be used to replace $\widehat{\beta}_{1}$. Then, the right hand side of Eq. (51) can be used to replace the summation term on the right hand side of Eq. (53). Consequently, we get the following relationship:

$$
\sum_{i=1}^{n} y_{i} x_{i}=\sum_{\xi=1}^{q} \sqrt{A_{b a l}^{2}(\xi)+S_{b a l}^{2}(\xi)+N_{b a l}^{2}(\xi)}
$$

At this point, all generic terms used in Eqs. (47a) and (47b) (i.e., terms associated with $\widehat{\beta}_{1}, n, x_{i}$, and $y_{i}$ ) can be replaced with corresponding wind tunnel model specific terms (i.e., terms associated with $\widehat{w}, q, A_{b a l}$, $S_{b a l}$, and $N_{b a l}$ ) by using the right hand sides of Eqs. (48), (50), (51), (52), and (54). Then, after simplifying and rearranging the result, we get the following final explicit equations for the confidence interval on the model weight: 


\section{EXPLICIT SOLUTION OF THE CONFIDENCE INTERVAL ON THE MODEL WEIGHT}

$$
\widehat{w}-t_{\alpha / 2,3 q-1} \cdot C(q) \leq w \leq \widehat{w}+t_{\alpha / 2,3 q-1} \cdot C(q)
$$

where the weight $\widehat{w}$ is given by Eq. (30) and the parameter $C(q)$ is defined as

$$
C(q)=\sqrt{\frac{E(q)-F(q)}{q \cdot[3 q-1]}}
$$

and the auxiliary parameters $E(q)$ and $F(q)$ are defined as

$$
\begin{aligned}
& E(q)=\sum_{\xi=1}^{q}\left[A_{b a l}^{2}(\xi)+S_{b a l}^{2}(\xi)+N_{b a l}^{2}(\xi)\right] \\
& F(q)=\frac{1}{q} \cdot\left[\sum_{\xi=1}^{q} \sqrt{A_{b a l}^{2}(\xi)+S_{b a l}^{2}(\xi)+N_{b a l}^{2}(\xi)}\right]^{2}
\end{aligned}
$$

One parameter in Eq. (55a) still needs to be specified before the confidence interval on the model weight can explicitly be calculated. It is the percentage point of the $t$-distribution $\left(t_{\alpha / 2,3 q-1}\right)$. This constant depends on (i) the confidence interval choice (e.g., $95 \%$ or $99 \%$ confidence interval) and (ii) the number of "wind-off" data points (i.e., q) that are used for the least squares fit associated with the model weight. The limiting case, i.e., the use of a single data point for the least squares fit, must be avoided. This case will not allow for the calculation of the confidence interval on the model weight as the confidence interval will be zero. This assertion can easily be proven "by inspection" because the following relationships apply:

$$
q=1 \quad \Longrightarrow \quad E(1)=F(1) \quad \Longrightarrow \quad C(1)=0
$$

The confidence interval is zero because the factor $C(1)$ is zero. Therefore, the number of data points needs to be greater than one in order to obtain a meaningful non-zero value for the confidence interval. Table 4 below lists the percentage points for different confidence interval choices and data point numbers.

Table 4: Percentage Points of the $t$ Distribution for $p=1$ (from Ref. [4], p. 515, Table A.3).

\begin{tabular}{|c|c|c|c|c|c|}
\hline CI & $\alpha$ & $\alpha / 2$ & $q$ & $\nu=3 q-1$ & $t_{\alpha / 2,3 q-1}$ \\
\hline \hline $95 \%$ & 0.05 & 0.025 & 3 & 8 & 2.306 \\
$95 \%$ & 0.05 & 0.025 & 5 & 14 & 2.145 \\
$95 \%$ & 0.05 & 0.025 & 10 & 29 & 2.045 \\
\hline $99 \%$ & 0.01 & 0.005 & 3 & 8 & 3.355 \\
$99 \%$ & 0.01 & 0.005 & 5 & 14 & 2.977 \\
$99 \%$ & 0.01 & 0.005 & 10 & 29 & 2.756 \\
\hline
\end{tabular}

Table 4 shows that already a total number of five data points (i.e., $q=5$ ) leads to percentage points of the $t$-distribution that are near the value of 2 for the $95 \%$ confidence interval and near the value of 3 for the $99 \%$ confidence interval. 


\section{Appendix 2: Upper Bound of the Model Weight Prediction Error}

It was shown in Eq. (30) that the least squares estimator of the model weight equals the arithmetic mean of the weights that are computed for each "wind-off" data point. Therefore, it is possible to perform an error analysis of the model weight prediction that (i) assesses contributions of the different balance load measurements to the total weight prediction error and (ii) defines an upper bound of the prediction error. The analysis of the model weight prediction error starts by expressing Eq. (30) in a slightly different format. The least squares estimator of the model weight can be described by the following equation:

$$
\widehat{w}=\frac{1}{q} \cdot \sum_{\xi=1}^{q} w^{\prime}(\xi) \quad \text { where } \quad w^{\prime}(\xi)=\sqrt{A_{b a l}^{2}(\xi)+S_{b a l}^{2}(\xi)+N_{b a l}^{2}(\xi)}
$$

The model's roll angle is often either $0^{\circ}$ or $180^{\circ}$ when "wind-off" data points are recorded. Then, the gravity vector's component in the side force direction of the balance axis system is zero. Therefore, we get:

$$
\gamma_{y} \approx 0 \quad \Longrightarrow \quad S_{b a l} \approx 0
$$

Now, after applying Eq. (58) in Eq. (57) and after dropping the "wind-off" data point index $\xi$ for simplicity, the summation term $w^{\prime}(\xi)$ defined in Eq. (57) becomes:

$$
w^{\prime}=\sqrt{A_{b a l}^{2}+N_{b a l}^{2}}
$$

Equation (59) assumes that both the axial and normal force measurements are "perfect." In reality, however, the prediction of balance loads is always associated with errors. These errors are primarily caused by load magnitude measurement and load alignment errors that occurred during the calibration of the balance. Therefore, the summation term defined in Eq. (59) has to be replaced by the sum of the "true" model weight and an associated weight prediction error. We get:

$$
w^{\prime} \Longrightarrow w^{\prime}+\Delta w^{\prime}
$$

Similarly, the balance loads need to be replaced by the sum of the unknown "true" load and the associated load prediction error. Then, we get for the axial and normal forces the relationships:

$$
\begin{aligned}
& A_{b a l} \quad \Longrightarrow \quad A_{b a l}+\Delta A_{b a l} \\
& N_{b a l} \quad \Longrightarrow \quad N_{b a l}+\Delta N_{b a l}
\end{aligned}
$$

Consequently, after replacing the loads in Eq. (59) by the right hand sides of Eqs. (61a) and (61b), the sum of the true model weight and the weight prediction error can be written as follows:

$$
w^{\prime}+\Delta w^{\prime}=\sqrt{\left[A_{b a l}+\Delta A_{b a l}\right]^{2}+\left[N_{b a l}+\Delta N_{b a l}\right]^{2}}
$$

Then, after expanding the brackets on the right hand side of Eq. (62), we get:

$$
w^{\prime}+\Delta w^{\prime}=\sqrt{\left[A_{b a l}^{2}+N_{b a l}^{2}\right]+\left[\Delta A_{b a l}^{2}+\Delta N_{b a l}^{2}\right]+2 \cdot\left[A_{b a l} \cdot \Delta A_{b a l}+N_{b a l} \cdot \Delta N_{b a l}\right]}
$$

We also know, after comparing the order of magnitude of terms on the right hand side of Eq. (63), that the following inequality and approximation are valid:

$$
\begin{gathered}
2 \cdot\left|[A_{b a l} \cdot \underbrace{\Delta A_{b a l}}_{1 \text { st order }}+N_{\text {bal }} \cdot \underbrace{\Delta N_{b a l}}_{1 \text { st order }}]\right| \gg \underbrace{\Delta A_{b a l}^{2}}_{2 n d \text { order }}+\underbrace{\Delta N_{b a l}^{2}}_{2 n d \text { order }} \\
\text { second order terms } \Longrightarrow \Delta A_{\text {bal }}^{2}+\Delta N_{\text {bal }}^{2} \approx 0
\end{gathered}
$$


In addition, the following abbreviation can be introduced:

$$
\text { first order terms } \Longrightarrow \epsilon=2 \cdot\left[A_{b a l} \cdot \Delta A_{b a l}+N_{b a l} \cdot \Delta N_{b a l}\right]
$$

Then, after using the relationships given in Eqs. (64a), (64b), and (64c), Eq. (63) becomes

$$
w^{\prime}+\Delta w^{\prime} \approx \sqrt{\left[A_{b a l}^{2}+N_{b a l}^{2}\right]+\epsilon}
$$

It is possible to simplify the right hand side of Eq. (65) by using a Taylor Series. In general, the Taylor Series approximation of a function in the neighborhood of an independent variable $\psi$ is defined as follows

$$
f(\psi+\epsilon) \approx f(\psi)+\frac{f^{\prime}(\psi)}{1 !} \cdot \epsilon+\frac{f^{\prime \prime}(\psi)}{2 !} \cdot \epsilon^{2}+\cdots
$$

where $\epsilon$ is a small value. Then, after comparing the right hand side of Eq. (65) with the left hand side of Eq. (66), we know that

$$
f(\psi+\epsilon)=\sqrt{\left[A_{b a l}^{2}+N_{b a l}^{2}\right]+\epsilon}
$$

Therefore, we need to find a Taylor Series expansion for the following function type:

$$
f(\psi)=\sqrt{\psi}
$$

where the first derivative of $f(\psi)$ is given by the following expression:

$$
f^{\prime}(\psi)=\frac{1}{2} \cdot \frac{1}{\sqrt{\psi}}
$$

Then, after inserting Eqs. (68a) and (68b) into the right hand side of Eq. (66) and after neglecting higher order terms by truncating the Taylor Series after the first derivative, we get:

$$
\sqrt{\psi+\epsilon} \approx \sqrt{\psi}+\frac{\epsilon}{2} \cdot \frac{1}{\sqrt{\psi}}
$$

We also know that

$$
\psi=A_{b a l}^{2}+N_{b a l}^{2}
$$

Then, after replacing $\psi$ in Eq. (69) by the right hand side of Eq. (70) and after comparing the result with the left hand side of Eq. (65), we get:

$$
w^{\prime}+\Delta w^{\prime} \approx \sqrt{\left[A_{b a l}^{2}+N_{b a l}^{2}\right]+\epsilon} \approx \sqrt{A_{b a l}^{2}+N_{b a l}^{2}}+\frac{\epsilon}{2} \cdot \frac{1}{\sqrt{A_{b a l}^{2}+N_{b a l}^{2}}}
$$

We also know, after rearranging Eq. (64c), that the following relationship is valid:

$$
\frac{\epsilon}{2}=A_{b a l} \cdot \Delta A_{b a l}+N_{b a l} \cdot \Delta N_{b a l}
$$

Then, after using the right hand side of Eq. (72) to replace the factor $\epsilon / 2$ in Eq. (71), we get:

$$
w^{\prime}+\Delta w^{\prime} \approx \underbrace{\sqrt{A_{b a l}^{2}+N_{b a l}^{2}}}_{\text {equals } w^{\prime}}+\frac{A_{b a l} \cdot \Delta A_{b a l}+N_{b a l} \cdot \Delta N_{b a l}}{\underbrace{\sqrt{A_{b a l}^{2}+N_{b a l}^{2}}}_{\text {equals } w^{\prime}}}
$$

We also know that the two square roots on the right hand side of Eq. (73) can be replaced by the left hand side of Eq. (59). Then, after simplifying the result, we get:

$$
w^{\prime}+\Delta w^{\prime} \approx w^{\prime}+\frac{A_{b a l} \cdot \Delta A_{b a l}+N_{b a l} \cdot \Delta N_{b a l}}{w^{\prime}}
$$


Now, a term can be identified in Eq. (74) that may be used to study the model weight prediction error. We get the following estimate of this error after simply comparing the left and right hand sides of Eq. (74):

$$
\Delta w^{\prime} \approx \frac{A_{b a l} \cdot \Delta A_{b a l}+N_{b a l} \cdot \Delta N_{b a l}}{w^{\prime}}
$$

It is possible to define an upper bound of the weight prediction error as the following inequality applies:

$$
\Delta w^{\prime} \approx \frac{A_{b a l} \cdot \Delta A_{b a l}+N_{b a l} \cdot \Delta N_{b a l}}{w^{\prime}} \leq \frac{\left|A_{b a l}\right| \cdot \Delta A_{b a l}+\left|N_{b a l}\right| \cdot \Delta N_{b a l}}{w^{\prime}}
$$

In addition, the following trigonometric relationships between the axial force, normal force, weight estimate, and the pitch angle of the model are valid:

$$
\begin{aligned}
& \left|A_{\text {bal }}\right| \approx w^{\prime} \cdot|\sin \mu| \\
& \left|N_{\text {bal }}\right| \approx w^{\prime} \cdot|\cos \mu|
\end{aligned}
$$

Finally, after using the right hand sides of Eqs. $(77 a)$ and $(77 b)$ to replace the absolute values of the forces on the right hand side of Eq. (76), we get the following inequality for the upper bound for the error:

\section{UPPER BOUND OF THE MODEL WEIGHT PREDICTION ERROR}

$$
\Delta w^{\prime} \leq \underbrace{\Delta A_{\text {bal }} \cdot|\sin \mu|}_{\text {axial force contribution }}+\underbrace{\Delta N_{\text {bal }} \cdot|\cos \mu|}_{\text {normal force contribution }}
$$

In general, the normal force capacity of most 6-component strain-gage balances is one order of magnitude larger than the axial force capacity. Then, assuming that errors are given in engineering units, the error associated with the normal force prediction is expected to be roughly one order of magnitude larger than the error associated with the axial force prediction because the electrical output range of a strain-gage is typically sized to match the related load range. This conclusion can be summarized as follows:

$$
\Delta N_{b a l} \approx 10 \cdot \Delta A_{b a l} \Longrightarrow \Delta N_{b a l} \gg \Delta A_{b a l}
$$

The pitch angle of the model also has an influence on the weight prediction error as trigonometric functions of the pitch angle are used in inequality (78). Table 5 below compares, for example, the sine and cosine values for the typical pitch angle range of a wind tunnel model.

Table 5: Sine and cosine values for a typical pitch angle range.

\begin{tabular}{|c|c|c|}
\hline$\mu$ & $\sin \mu$ & $\cos \mu$ \\
\hline \hline $0^{\circ}$ & 0.000 & 1.000 \\
$5^{\circ}$ & 0.087 & 0.996 \\
$10^{\circ}$ & 0.174 & 0.985 \\
$20^{\circ}$ & 0.342 & 0.940 \\
\hline
\end{tabular}

Therefore, after combining inequality (79) with the expected values of the sine and cosine functions for the typical pitch angle range of a wind tunnel model, we get the following inequality:

$$
\Delta N_{b a l} \cdot|\cos \mu| \gg \Delta A_{b a l} \cdot|\sin \mu|
$$

Finally, after comparing inequality (80) with inequality (78), it is concluded that the error associated with the normal force prediction, i.e., $\Delta N_{b a l}$, is by far the largest contributor to the error associated with the model weight prediction over the typical pitch angle range of a wind tunnel model. This important conclusion can be illustrated by a numerical example. Let us assume that (i) the model is pitched to $20^{\circ}$ and that (ii) the normal force error $\Delta N_{b a l}$ is ten times as large as the axial force error $\Delta A_{b a l}$. Then, after evaluating all terms on the right hand side of inequality (78) and after simplifying the result, we see that the normal force contribution is $96.5 \%$ of the upper bound of the model weight prediction error. 


\section{Appendix 3: Explicit Solution of the Center of Gravity Coordinates}

An explicit solution of the center of gravity coordinates can be obtained after applying Cramer's Rule to the $3 \times 3$ linear system of equations that is defined in Eq. (37a) (see, e.g., Ref. [5], pp. 323-324, for a description of Cramer's Rule). First, the coefficients of matrix $\widehat{\mathbf{A}}^{\mathbf{T}} \widehat{\mathbf{A}}$ and the components of vector $\widehat{\mathbf{A}}^{\mathbf{T}} \widehat{\mathbf{B}}$ have to be determined by inspection. The following coefficients of matrix $\widehat{\mathbf{A}}^{\mathbf{T}} \widehat{\mathbf{A}}$ are obtained:

$$
\widehat{\mathbf{A}}^{\mathbf{T}} \widehat{\mathbf{A}}=\left[\begin{array}{lll}
u_{11} & u_{12} & u_{13} \\
u_{21} & u_{22} & u_{23} \\
u_{31} & u_{32} & u_{33}
\end{array}\right]
$$

where $u_{11}, u_{12}, \cdots, u_{33}$ are defined as follows:

$$
\begin{aligned}
& u_{11}=\widehat{w}^{2} \cdot \sum_{\xi=1}^{q}\left[\gamma_{y}(\xi)^{2}+\gamma_{z}(\xi)^{2}\right] \\
& u_{22}=\widehat{w}^{2} \cdot \sum_{\xi=1}^{q}\left[\gamma_{z}(\xi)^{2}+\gamma_{x}(\xi)^{2}\right] \\
& u_{33}=\widehat{w}^{2} \cdot \sum_{\xi=1}^{q}\left[\gamma_{x}(\xi)^{2}+\gamma_{y}(\xi)^{2}\right] \\
& u_{12}=u_{21}=-\widehat{w}^{2} \cdot \sum_{\xi=1}^{q}\left[\gamma_{x}(\xi) \cdot \gamma_{y}(\xi)\right] \\
& u_{13}=u_{31}=-\widehat{w}^{2} \cdot \sum_{\xi=1}^{q}\left[\gamma_{z}(\xi) \cdot \gamma_{x}(\xi)\right] \\
& u_{23}=u_{32}=-\widehat{w}^{2} \cdot \sum_{\xi=1}^{q}\left[\gamma_{y}(\xi) \cdot \gamma_{z}(\xi)\right]
\end{aligned}
$$

Similarly, the following components are obtained for vector $\widehat{\mathbf{A}}^{\mathbf{T}} \widehat{\mathbf{B}}$ :

$$
\widehat{\mathbf{A}}^{\mathbf{T}} \widehat{\mathbf{B}}=\left[\begin{array}{l}
v_{1} \\
v_{2} \\
v_{3}
\end{array}\right]
$$

where $v_{1}, v_{2}$, and $v_{3}$ are defined as follows:

$$
\begin{aligned}
v_{1} & =\widehat{w} \cdot \sum_{\xi=1}^{q}\left[Y_{b a l}(\xi) \cdot \gamma_{y}(\xi)-P_{b a l}(\xi) \cdot \gamma_{z}(\xi)\right] \\
v_{2} & =\widehat{w} \cdot \sum_{\xi=1}^{q}\left[R_{b a l}(\xi) \cdot \gamma_{z}(\xi)-Y_{b a l}(\xi) \cdot \gamma_{x}(\xi)\right] \\
v_{3} & =\widehat{w} \cdot \sum_{\xi=1}^{q}\left[P_{b a l}(\xi) \cdot \gamma_{x}(\xi)-R_{b a l}(\xi) \cdot \gamma_{y}(\xi)\right]
\end{aligned}
$$

The coefficients $u_{11}, u_{12}, \ldots, u_{33}$ and the components $v_{1}, v_{2}, v_{3}$ describe the left and right hand sides of the $3 \times 3$ linear system of equations that is defined in Eq. (37a). All these values have the factor $\widehat{w}$ in

common. Therefore, they can be divided by $\widehat{w}$ without changing the solution of the linear system, i.e., of the center of gravity coordinates. Then, the coefficients $u_{11}, \ldots, u_{33}$ are replaced by $r_{11}, \ldots, r_{33}$ :

$$
r_{11}=\widehat{w} \cdot \sum_{\xi=1}^{q}\left[\gamma_{y}(\xi)^{2}+\gamma_{z}(\xi)^{2}\right]
$$




$$
\begin{aligned}
& r_{22}=\widehat{w} \cdot \sum_{\xi=1}^{q}\left[\gamma_{z}(\xi)^{2}+\gamma_{x}(\xi)^{2}\right] \\
& r_{33}=\widehat{w} \cdot \sum_{\xi=1}^{q}\left[\gamma_{x}(\xi)^{2}+\gamma_{y}(\xi)^{2}\right] \\
& r_{12}=r_{21}=-\widehat{w} \cdot \sum_{\xi=1}^{q}\left[\gamma_{x}(\xi) \cdot \gamma_{y}(\xi)\right] \\
& r_{13}=r_{31}=-\widehat{w} \cdot \sum_{\xi=1}^{q}\left[\gamma_{z}(\xi) \cdot \gamma_{x}(\xi)\right] \\
& r_{23}=r_{32}=-\widehat{w} \cdot \sum_{\xi=1}^{q}\left[\gamma_{y}(\xi) \cdot \gamma_{z}(\xi)\right]
\end{aligned}
$$

Similarly, the components $v_{1}, v_{2}, v_{3}$ are replaced by $\rho_{1}, \rho_{2}, \rho_{3}$ :

$$
\begin{aligned}
\rho_{1} & =\sum_{\xi=1}^{q}\left[Y_{b a l}(\xi) \cdot \gamma_{y}(\xi)-P_{b a l}(\xi) \cdot \gamma_{z}(\xi)\right] \\
\rho_{2} & =\sum_{\xi=1}^{q}\left[R_{b a l}(\xi) \cdot \gamma_{z}(\xi)-Y_{b a l}(\xi) \cdot \gamma_{x}(\xi)\right] \\
\rho_{3} & =\sum_{\xi=1}^{q}\left[P_{b a l}(\xi) \cdot \gamma_{x}(\xi)-R_{b a l}(\xi) \cdot \gamma_{y}(\xi)\right]
\end{aligned}
$$

Finally, after applying Cramer's Rule, we get an explicit solution of the center of gravity coordinates:

\section{EXPLICIT SOLUTION OF THE CENTER OF GRAVITY COORDINATES}

$$
\begin{aligned}
& \widehat{d_{x}}=R_{1} / R \\
& \widehat{d}_{y}=R_{2} / R \\
& \widehat{d}_{z}=R_{3} / R
\end{aligned}
$$

where the four determinates $R, R_{1}, R_{2}$, and $R_{3}$ are defined as

$$
\begin{aligned}
R & =\left|\begin{array}{lll}
r_{11} & r_{12} & r_{13} \\
r_{21} & r_{22} & r_{23} \\
r_{31} & r_{32} & r_{33}
\end{array}\right| \\
R_{1} & =\left|\begin{array}{lll}
\rho_{1} & r_{12} & r_{13} \\
\rho_{2} & r_{22} & r_{23} \\
\rho_{3} & r_{32} & r_{33}
\end{array}\right| \\
R_{2} & =\left|\begin{array}{lll}
r_{11} & \rho_{1} & r_{13} \\
r_{21} & \rho_{2} & r_{23} \\
r_{31} & \rho_{3} & r_{33}
\end{array}\right| \\
R_{3} & =\left|\begin{array}{lll}
r_{11} & r_{12} & \rho_{1} \\
r_{21} & r_{22} & \rho_{2} \\
r_{31} & r_{32} & \rho_{3}
\end{array}\right|
\end{aligned}
$$




\section{Appendix 4: Confidence Intervals on the Center of Gravity Coordinates}

The center of gravity coordinates of the wind tunnel model were introduced as regression coefficients in an earlier section of the paper. These coefficients were obtained after fitting the predicted moments of a strain-gage balance as a function of (i) related model attitude measurements and (ii) the least squares estimator of the model weight. The reporting of the center of gravity coordinates becomes more complete if confidence intervals on the coordinates are estimated. Therefore, explicit equations for the confidence intervals on the center of gravity coordinates are discussed in this section. These equations were derived from more general relationships that are reported in the literature (see Ref. [4], pp. 93-94). This approach was selected as a derivation of the general equations of the confidence intervals goes beyond the scope of the present paper. First, the general equations of the confidence interval on a regression coefficient are developed using information that is provided in the literature. Then, it is shown how these general equations of the confidence intervals can be applied to the center of gravity coordinates. For simplicity, it is also assumed that the least squares estimator of the model weight, i.e., $\widehat{w}$, can be treated like a constant in the regression model of the center of gravity coordinates.

The discussion of the general equations of the confidence interval on a regression coefficient starts with a generic "multiple" linear regression model that is given in the literature. This model can be described as follows (from Ref. [4], Eq. (3.2)):

$$
y=\beta_{0}+\beta_{1} \cdot x_{1}+\beta_{2} \cdot x_{2}+\cdots+\beta_{k} \cdot x_{k}+\epsilon
$$

where $x$ is the regressor variable, $y$ is the response variable, $\beta_{0}, \ldots, \beta_{k}$ are the regression coefficients, and $\epsilon$ is a small quantity. We know from Eq. (32) that the regression model associated with the center of gravity coordinates has no intercept (i.e., $\beta_{0}=0$ ), and consists of three regression coefficients (i.e., $k=3$ ). Therefore, after also dropping the small quantity $\epsilon$, Eq. (89a) can be written as follows:

$$
y=\beta_{1} \cdot x_{1}+\beta_{2} \cdot x_{2}+\beta_{3} \cdot x_{3}
$$

Furthermore, it is assumed that a total of $n$ observations, i.e., $\left(y_{i}, x_{i}\right), i=1,2, \ldots, n$, are to be used to determine the least squares estimators $\widehat{\beta}_{1}, \widehat{\beta}_{2}, \widehat{\beta}_{3}$ of the coefficients $\beta_{1}, \beta_{2}, \beta_{3}$ of Eq. (89b). Then, Eq. (89b) can be expressed for each observation with index $i$ by using the following equation (see also Ref. [4], Eq. (3.8)):

$$
y_{i}=\beta_{1} \cdot x_{i 1}+\beta_{2} \cdot x_{i 2}+\beta_{3} \cdot x_{i 3} ; 1 \leq i \leq n
$$

Reference [4] constructs the confidence interval on the three regression coefficients $\beta_{1}, \beta_{2}, \beta_{3}$ that are given in Eq. (90) by making the normality assumption on the errors of the least squares fit. Then, the $100(1-\alpha)$ percent confidence interval on the coefficients $\beta_{j}(j=1,2,3)$ of the model is defined as (from Ref. [4], Eqs. (3.45)):

\section{CONFIDENCE INTERVALS ON THE REGRESSION COEFFICIENTS} (... taken from Ref. [4], Eqs. (3.16), (3.17), (3.18), (3.45) ...)

$$
\widehat{\beta}_{j}-t_{\alpha / 2, n-p} \cdot \sqrt{\widehat{\sigma}^{2} C_{j j}} \leq \beta_{j} \leq \widehat{\beta}_{j}+t_{\alpha / 2, n-p} \cdot \sqrt{\widehat{\sigma}^{2} C_{j j}}
$$

where $C_{j j}$ is the $j$ th diagonal element of $\left[\mathbf{X}^{\prime} \mathbf{X}\right]^{-1}$ and the estimate of the error variance $\widehat{\sigma}^{2}$ is defined as follows

$$
\widehat{\sigma}^{2}=\frac{S S_{\text {Res }}}{n-p}=\frac{1}{n-p} \cdot\left[\mathbf{y}^{\prime} \mathbf{y}-\widehat{\boldsymbol{\beta}}^{\prime} \mathbf{X}^{\prime} \mathbf{y}\right]
$$

Equations (91a) and (91b) can be used to construct the confidence intervals on the center of gravity coordinates after the generic nomenclature used in Ref. [4] is "translated" to the specific nomenclature 
that is used in the present paper for the calculation of the least squares estimators of the center of gravity coordinates. Table 6 below summarizes analogies between the nomenclature of Ref. [4] and the nomenclature used in the present paper that were identified after comparing terms of Eq. (32) with terms of Eq. (90).

Table 6: Comparison of Nomenclature used for Center of Gravity Coordinate Determination.

\begin{tabular}{|c|c|c|}
\hline Reference $[4]$ & present paper & Comments \\
\hline \hline$\beta_{1}, \beta_{2}, \beta_{3}$, & $d_{x}, d_{y}, d_{z}$ & regression coefficients; c.g. coordinates \\
\hline$\widehat{\beta}_{1}, \widehat{\beta}_{2}, \widehat{\beta}_{3}$, & $\widehat{d_{x}}, \widehat{d_{y}}, \widehat{d}_{z}$ & least squares estimators; Eqs. (87a), (87b), (87c) \\
\hline$n$ & $3 q$ & total number of "observations" \\
\hline$p$ & 3 & total number of regression model parameter \\
\hline$x_{11}, x_{12}, \ldots, x_{n 3}$ & $0,+\gamma_{z}(1) \cdot \widehat{w}, \ldots, 0$ & coefficient values of model matrix \\
\hline$y_{1}, y_{2}, \ldots, y_{n}$ & $R_{b a l}(1), P_{b a l}(1), \ldots, Y_{b a l}(q)$ & response variable values \\
\hline
\end{tabular}

In addition, because generic vectors and matrices are used in Eq. (91b), it is necessary to identify corresponding vectors and matrices that were used in the present paper for the calculation of the center of gravity coordinates. Table 7 below summarizes analogies between vectors and matrices used in Ref. [4] and vectors and matrices used in the present paper that were identified after comparing Eq. (3.13) of Ref. [4] with Eqs. (35a), (35b), and (37b) of the present paper.

Table 7: Comparison of Vector and Matrix Nomenclature.

\begin{tabular}{|c|c|c|c|}
\hline Reference [4] & present paper & Rows $\times$ Columns & Comments \\
\hline \hline $\mathbf{y}$ & $\widehat{\mathbf{B}}$ & $3 q \times 1$ & Eq. (35b) \\
\hline $\mathbf{y}^{\prime}$ & $\widehat{\mathbf{B}}^{\mathbf{T}}$ & $1 \times 3 q$ & transpose matrix \\
\hline $\mathbf{X}$ & $\widehat{\mathbf{A}}$ & $3 q \times 3$ & Eq. (35a) \\
\hline $\mathbf{X}^{\prime}$ & $\widehat{\mathbf{A}}^{\mathbf{T}}$ & $3 \times 3 q$ & transpose matrix \\
\hline$\widehat{\beta}$ & $\widehat{\mathbf{X}}$ & $3 \times 1$ & Eqs. (37b), (87a), (87b), (87c) \\
\hline$\widehat{\boldsymbol{\beta}}^{\prime}$ & $\widehat{\mathbf{X}}^{\mathbf{T}}$ & $1 \times 3$ & transpose matrix \\
\hline
\end{tabular}

Now it is possible to identify the remaining terms in Eqs. $(91 a)$ and $(91 b)$. First, $C_{j j}$, i.e., the $j$ th diagonal element of the matrix $\left[\mathbf{X}^{\prime} \mathbf{X}\right]^{-1}$ needs to be obtained. We know, after looking at Table 7 , that the following relationship applies:

$$
\mathbf{X}^{\prime} \mathbf{X}=\widehat{\mathbf{A}}^{\mathbf{T}} \widehat{\mathbf{A}}=\left[\begin{array}{lll}
u_{11} & u_{12} & u_{13} \\
u_{21} & u_{22} & u_{23} \\
u_{31} & u_{32} & u_{33}
\end{array}\right]
$$

where the coefficients $u_{11}, u_{12}, \ldots, u_{33}$ are defined in Eqs. $(82 a)$ to $(82 f)$. The unknown coefficients of the inverse matrix of $\left[\mathbf{X}^{\prime} \mathbf{X}\right]$ can be expressed as follows:

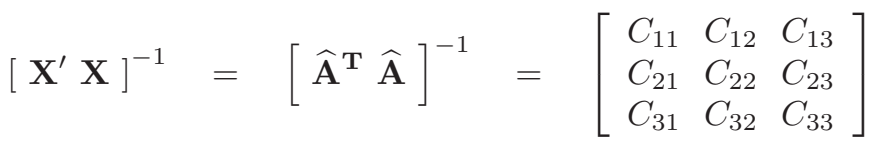

We also know that the product of a square matrix with its inverse equals the identify matrix. Therefore, we can write:

$$
\left[\begin{array}{ll}
\widehat{\mathbf{A}}^{\mathbf{T}} & \widehat{\mathbf{A}}
\end{array}\right] \cdot\left[\begin{array}{ll}
\widehat{\mathbf{A}}^{\mathbf{T}} & \widehat{\mathbf{A}}
\end{array}\right]^{-1}=\mathbf{I}=\left[\begin{array}{lll}
1 & 0 & 0 \\
0 & 1 & 0 \\
0 & 0 & 1
\end{array}\right]
$$


Each coefficient of the inverse matrix can easily be found by using Cramer's Rule. It is only required to identify the related three $3 \times 3$ determinates that have to be constructed by using the coefficients of matrix $\widehat{\mathbf{A}}^{\mathbf{T}} \widehat{\mathbf{A}}$ and the coefficients of the identity matrix $\mathbf{I}$. Then, by inspection, we get for $C_{11}, C_{22}$, and $C_{33}$

$$
\begin{aligned}
& C_{11}=U_{1} / U \\
& C_{22}=U_{2} / U \\
& C_{33}=U_{3} / U
\end{aligned}
$$

where the four determinates $U, U_{1}, U_{2}$, and $U_{3}$ are defined as

$$
\begin{gathered}
U=\left|\begin{array}{lll}
u_{11} & u_{12} & u_{13} \\
u_{21} & u_{22} & u_{23} \\
u_{31} & u_{32} & u_{33}
\end{array}\right| \\
U_{1}=\left|\begin{array}{lll}
1 & u_{12} & u_{13} \\
0 & u_{22} & u_{23} \\
0 & u_{32} & u_{33}
\end{array}\right|=u_{22} u_{33}-u_{23} u_{32} \\
U_{2}=\left|\begin{array}{lll}
u_{11} & 0 & u_{13} \\
u_{21} & 1 & u_{23} \\
u_{31} & 0 & u_{33}
\end{array}\right|=u_{11} u_{33}-u_{13} u_{31} \\
U_{3}=\left|\begin{array}{lll}
u_{11} & u_{12} & 0 \\
u_{21} & u_{22} & 0 \\
u_{31} & u_{32} & 1
\end{array}\right|=u_{11} u_{22}-u_{12} u_{21}
\end{gathered}
$$

and $u_{11}, u_{12}, \ldots, u_{33}$ are defined in Eqs. (82a) to $(82 f)$. The term $\mathbf{y}^{\prime} \mathbf{y}-\widehat{\boldsymbol{\beta}}^{\prime} \mathbf{X}^{\prime} \mathbf{y}$ of Eq. (91b) still needs to be identified before an explicit solution of the confidence intervals on the center of gravity coordinates can be assembled. From Table 7 we get:

$$
\mathbf{y}^{\prime} \mathbf{y}=\widehat{\mathbf{B}}^{\mathbf{T}} \widehat{\mathbf{B}}
$$

We also know after looking at Eqs. (33b) and (35b) that the following relationship applies:

$$
\begin{aligned}
\widehat{\mathbf{B}}^{\mathbf{T}} \widehat{\mathbf{B}} & =\widehat{\mathbf{L}}^{\mathbf{T}}(1) \widehat{\mathbf{L}}(1)+\cdots+\widehat{\mathbf{L}}^{\mathbf{T}}(q) \widehat{\mathbf{L}}(q) \\
& =R_{b a l}^{2}(1)+P_{b a l}^{2}(1)+Y_{b a l}^{2}(1)+\cdots+R_{b a l}^{2}(q)+P_{b a l}^{2}(q)+Y_{b a l}^{2}(q) \\
& =\sum_{\xi=1}^{q}\left[R_{b a l}^{2}(\xi)+P_{b a l}^{2}(\xi)+Y_{b a l}^{2}(\xi)\right]
\end{aligned}
$$

From Table 7 we also know that the following relationship is valid:

$$
\widehat{\beta}^{\prime} \mathbf{X}^{\prime} \mathbf{y}=\widehat{\mathbf{X}}^{\mathbf{T}} \widehat{\mathbf{A}}^{\mathbf{T}} \widehat{\mathbf{B}}
$$

In addition, after taking the transpose of the left hand side of Eq. (37b), we get:

$$
\left[\widehat{\mathbf{X}}^{\mathbf{T}}\right]_{1 \times 3}=\left[\begin{array}{lll}
\widehat{d}_{x} & \widehat{d}_{y} & \widehat{d}_{z}
\end{array}\right]
$$

We also know from Eq. (83) that the following relationship applies:

$$
\left[\widehat{\mathbf{A}}^{\mathbf{T}} \widehat{\mathbf{B}}\right]_{3 \times 1}=\left[\begin{array}{l}
v_{1} \\
v_{2} \\
v_{3}
\end{array}\right]
$$


Finally, after computing the scalar product of the right hand sides of Eqs. (99a) and (99b), we get:

$$
\widehat{\mathbf{X}}^{\mathbf{T}} \widehat{\mathbf{A}}^{\mathbf{T}} \widehat{\mathbf{B}}=\widehat{d}_{x} \cdot v_{1}+\widehat{d}_{y} \cdot v_{2}+\widehat{d}_{z} \cdot v_{3}
$$

At this point, all generic terms used in Eqs. (91a) and (91b) have been identified. Then, after using the contents of Table 6 in combination with Eqs. (94a), (94b), (94c), (97), and (100), we get the following final explicit equations for the confidence intervals on the center of gravity coordinates:

\section{EXPLICIT SOLUTION OF THE CONFIDENCE INTERVALS ON THE CENTER OF GRAVITY COORDINATES}

$$
\begin{aligned}
& \widehat{d}_{x}-t_{\alpha / 2,3 q-3} \cdot C_{x} \leq d_{x} \leq \widehat{d}_{x}+t_{\alpha / 2,3 q-3} \cdot C_{x} \\
& \widehat{d}_{y}-t_{\alpha / 2,3 q-3} \cdot C_{y} \leq d_{y} \leq \widehat{d}_{y}+t_{\alpha / 2,3 q-3} \cdot C_{y} \\
& \widehat{d}_{z}-t_{\alpha / 2,3 q-3} \cdot C_{z} \leq d_{z} \leq \widehat{d}_{z}+t_{\alpha / 2,3 q-3} \cdot C_{z}
\end{aligned}
$$

where the standard errors $C_{x}, C_{y}$, and $C_{z}$ of the coordinates are defined as

$$
\begin{aligned}
& C_{x}=\sqrt{\widehat{\sigma}^{2} C_{11}} \\
& C_{y}=\sqrt{\widehat{\sigma}^{2} C_{22}} \\
& C_{z}=\sqrt{\widehat{\sigma}^{2} C_{33}}
\end{aligned}
$$

and the estimate of the error variance $\widehat{\sigma}^{2}$ is defined as

$$
\begin{aligned}
\widehat{\sigma}^{2} & =\frac{1}{3 q-3} \cdot[G(q)-H(q)] \\
G(q) & =\sum_{\xi=1}^{q}\left[R_{b a l}^{2}(\xi)+P_{b a l}^{2}(\xi)+Y_{b a l}^{2}(\xi)\right] \\
H(q) & =\widehat{d}_{x} \cdot v_{1}+\widehat{d}_{y} \cdot v_{2}+\widehat{d}_{z} \cdot v_{3}
\end{aligned}
$$

Table 8 below lists equations that define the omitted parameters that are used in Eqs. (101a) to (101i).

Table 8: List of Equation Numbers.

\begin{tabular}{|c|c|c|c|c|c|c|c|c|c|}
\hline Parameter & $\widehat{d}_{x}$ & $\widehat{d}_{y}$ & $\widehat{d}_{z}$ & $C_{11}$ & $C_{22}$ & $C_{33}$ & $v_{1}$ & $v_{2}$ & $v_{3}$ \\
\hline \hline Equation & $(87 a)$ & $(87 b)$ & $(87 c)$ & $(94 a)$ & $(94 b)$ & $(94 c)$ & $(84 a)$ & $(84 b)$ & $(84 c)$ \\
\hline
\end{tabular}

One parameter in Eqs. (101a), (101b), (101c) still needs to be specified before the confidence intervals on the center of gravity coordinates can explicitly be calculated. It is the percentage point of the $t$-distribution (i.e., $t_{\alpha / 2,3 q-3}$ ). This constant depends on (i) the confidence interval choice (e.g., $95 \%$ or $99 \%$ confidence interval) and (ii) the number of data points (i.e., q) that are used for the least squares fit associated with the center of gravity coordinates. Table 9 below lists the percentage points for different confidence interval choices and data point numbers. 
Table 9: Percentage Points of the $t$ Distribution for $p=3$ (from Ref. [4], p. 515, Table A.3).

\begin{tabular}{|c|c|c|c|c|c|}
\hline $\mathrm{CI}$ & $\alpha$ & $\alpha / 2$ & $q$ & $\nu=3 q-3$ & $t_{\alpha / 2,3 q-3}$ \\
\hline \hline $95 \%$ & 0.05 & 0.025 & 3 & 6 & 2.447 \\
$95 \%$ & 0.05 & 0.025 & 5 & 12 & 2.179 \\
$95 \%$ & 0.05 & 0.025 & 10 & 27 & 2.052 \\
\hline $99 \%$ & 0.01 & 0.005 & 3 & 6 & 3.707 \\
$99 \%$ & 0.01 & 0.005 & 5 & 12 & 3.055 \\
$99 \%$ & 0.01 & 0.005 & 10 & 27 & 2.771 \\
\hline
\end{tabular}

Table 9 shows that at least five data points (i.e., $q=5$ ) are needed in order to obtain percentage points of the $t$-distribution that are near the value of 2 for the $95 \%$ confidence interval and near the value of 3 for the $99 \%$ confidence interval. Therefore, the authors recommend to use at least five data points for the least squares fit that is associated with the regression analysis associated with the determination of both the model weight and center of gravity coordinates. 


\section{Appendix 5: General Error Analysis of the Model Weight Prediction}

An estimate of the upper bound of the wind tunnel model weight prediction error was derived in App. 2 of the paper. A more general error analysis of the model weight prediction can be performed by applying a well-known error propagation formula from the literature to Eq. (30). This formula estimates the variance in the model weight prediction (a discussion of the error propagation formula is given, e.g., in Ref. [8], p. 49).

The general analysis of the model weight prediction error and the application of the error propagation formula starts by expressing Eq. (30) in a slightly different format. The least squares estimator of the model weight can be described by the following set of equations:

$$
\widehat{w}=\frac{1}{q} \cdot \sum_{\xi=1}^{q} w^{\prime}(\xi)
$$

where

$$
w^{\prime}(\xi)=\sqrt{A_{b a l}^{2}(\xi)+S_{b a l}^{2}(\xi)+N_{b a l}^{2}(\xi)}
$$

It can be concluded from the above equations that the final model weight estimate is a function of the individual axial force, side force, and normal force measurements of each data point that is used to compute the model weight estimate. This conclusion can be summarized as follows:

$$
\widehat{w}=\mathcal{F}\left[A_{b a l}(1), \ldots, A_{b a l}(q), S_{b a l}(1), \ldots, S_{b a l}(q), N_{b a l}(1), \ldots, N_{b a l}(q)\right]
$$

Now, it is possible to apply the error propagation formula from the literature to Eq. (103). The error propagation formula is defined for a multivariate function $Y$ where $X_{1}, \ldots, X_{\phi}$ are a set of independent variables. This characteristic of function $Y$ can be summarized as follows:

$$
Y=\mathcal{F}\left[X_{1}, X_{2}, \ldots, X_{\phi}\right]
$$

The error propagation formula is given by the following formula (taken from Ref. [8], p. 49, Eq. (3.3)):

$$
\sigma^{2}(Y)=\left(\frac{\partial Y}{\partial X_{1}}\right)^{2} \cdot \sigma^{2}\left(X_{1}\right)+\cdots+\left(\frac{\partial Y}{\partial X_{\phi}}\right)^{2} \cdot \sigma^{2}\left(X_{\phi}\right)
$$

The error propagation formula can be applied to Eq. (103) after comparing the nomenclature used in Eq. (103) with the nomenclature used in Eq. (104a). Table 10 below lists corresponding symbols.

Table 10: Comparison of Nomenclature used by the Error Propagation Formula.

\begin{tabular}{|c|c|}
\hline Nomenclature used in Eq. (104a) & Nomenclature used in Eq. (103) \\
\hline \hline$Y$ & $\widehat{w}$ \\
\hline$X_{1}, X_{2}, \ldots, X_{\phi}$ & $A_{b a l}(1), \ldots, A_{b a l}(q), S_{b a l}(1), \ldots, S_{b a l}(q), N_{b a l}(1), \ldots, N_{b a l}(q)$ \\
\hline$\phi$ & $3 q$ \\
\hline
\end{tabular}

Then, after replacing the generic symbols used Eq. (104b) with the symbols listed in the second column of Table 10, we get the following error propagation formula for the model weight estimate

$$
\sigma^{2}(\widehat{w})=\left(\frac{\partial \widehat{w}}{\partial A_{b a l}(1)}\right)^{2} \cdot \sigma^{2}\left(A_{b a l}(1)\right)+\cdots+\left(\frac{\partial \widehat{w}}{\partial N_{b a l}(q)}\right)^{2} \cdot \sigma^{2}\left(N_{b a l}(q)\right)
$$

where $\sigma^{2}(\widehat{w}), \sigma^{2}\left(A_{b a l}\right), \sigma^{2}\left(S_{b a l}\right)$, and $\sigma^{2}\left(N_{b a l}\right)$ are the variances in the model weight, axial force, side force, and normal force predictions. It is reasonable to assume that the variances in the axial force prediction are 
identical for all "wind-off" data points. The same assumption can be applied to the side force and normal force predictions. These conclusions can be expressed as follows:

$$
\begin{aligned}
& \sigma\left(A_{b a l}(1)\right)=\sigma\left(A_{b a l}(2)\right)=\cdots=\sigma\left(A_{b a l}(q)\right)=\text { const. }=\sigma(A) \\
& \sigma\left(S_{b a l}(1)\right)=\sigma\left(S_{b a l}(2)\right)=\cdots=\sigma\left(S_{b a l}(q)\right)=\text { const. }=\sigma(S) \\
& \sigma\left(N_{b a l}(1)\right)=\sigma\left(N_{b a l}(2)\right)=\cdots=\sigma\left(N_{b a l}(q)\right)=\text { const. }=\sigma(N)
\end{aligned}
$$

Partial derivatives used in Eq. (105) are obtained after applying the chain rule to Eq. (102a) and (102b). Then, we get:

$$
\begin{aligned}
& \frac{\partial \widehat{w}}{\partial A_{b a l}(\xi)}=\frac{A_{b a l}(\xi)}{q \cdot w^{\prime}(\xi)} \\
& \frac{\partial \widehat{w}}{\partial S_{b a l}(\xi)}=\frac{S_{b a l}(\xi)}{q \cdot w^{\prime}(\xi)} \\
& \frac{\partial \widehat{w}}{\partial N_{b a l}(\xi)}=\frac{N_{b a l}(\xi)}{q \cdot w^{\prime}(\xi)}
\end{aligned}
$$

Finally, after using the right hand sides of Eqs. $(106 a), \ldots,(107 c)$ to replace related terms in Eq. (105), we get the following relationship for the variance in the model weight prediction:

\section{VARIANCE IN THE MODEL WEIGHT PREDICTION}

$$
\sigma^{2}(\widehat{w})=\frac{1}{q^{2}} \sum_{\xi=1}^{q}\left[\left(\frac{A_{b a l}(\xi)}{w^{\prime}(\xi)}\right)^{2} \sigma^{2}(A)+\left(\frac{S_{b a l}(\xi)}{w^{\prime}(\xi)}\right)^{2} \sigma^{2}(S)+\left(\frac{N_{b a l}(\xi)}{w^{\prime}(\xi)}\right)^{2} \sigma^{2}(N)\right]
$$

The formula of the variance in the model weight prediction, i.e., Eq. (108), can significantly be simplified by taking subject matter knowledge about both wind tunnel test procedures and strain-gage balances into account. The roll angle of the wind tunnel model, for example, is often either $0^{\circ}$ or $180^{\circ}$ when "wind-off" data points are recorded. Consequently, the gravitational acceleration has no component in the direction of the side force. This conclusion can be summarized as follows:

$$
S_{b a l}(\xi) \approx 0
$$

We also know that the contribution of the normal force to the model weight estimate is significantly larger than the contribution of the axial force because (i) the primary sensitivity of the normal force of a typical strain-gage balance is one order of magnitude larger that the primary sensitivity of the axial force and (ii) the pitch angle of the wind tunnel model is in most cases less than $20^{\circ}$ when "wind-off" data points are recorded (see also the related discussion in App. 2). Therefore, the following inequality applies:

$$
\left|N_{b a l}\right| \gg\left|A_{b a l}\right| \quad \text { for } \quad|\mu|<20^{\circ}
$$

Consequently, the weight estimate $w^{\prime}(\xi)$ defined in Eq. (102b) for each individual "wind-off" data point with index $\xi$ can be approximated by using the relationship:

$$
w^{\prime}(\xi)=\sqrt{A_{b a l}^{2}(\xi)+S_{b a l}^{2}(\xi)+N_{b a l}^{2}(\xi)} \Longrightarrow\left\{\begin{array}{c}
S_{b a l}(\xi) \approx 0 \\
N_{b a l}^{2}(\xi) \gg A_{b a l}^{2}(\xi)
\end{array}\right\} \Longrightarrow w^{\prime}(\xi) \approx N_{b a l}(\xi)
$$

In addition, the variance in the normal force measurement is expected to be significantly larger than the variance in the axial force measurement because, as mentioned earlier, the primary sensitivity of the normal 
force of a typical strain-gage balance is one order of magnitude larger that the primary sensitivity of the axial force. This conclusion can be summarized as follows:

$$
\sigma(N) \gg \sigma(A)
$$

Then, after using the relationships given in Eqs. (109a), .., (109d) in Eq. (108), the following approximation of the variance in the model weight prediction is obtained:

$$
\sigma^{2}(\widehat{w}) \approx \frac{1}{q^{2}} \cdot \sum_{\xi=1}^{q} \sigma^{2}(N)
$$

Equation (110) can be simplified further because we know from Eq. (106c) that the variance in the normal force measurement is a constant. Therefore, the following relationship is valid:

$$
\sum_{\xi=1}^{q} \sigma^{2}(N)=q \cdot \sigma^{2}(N)
$$

Finally, after using the right hand side of Eq. (111) to replace the summation term on the right hand side of Eq. (110), we get the following approximation of the variance in the model weight prediction:

\section{APPROXIMATION OF THE VARIANCE IN THE MODEL WEIGHT PREDICTION}

$$
\sigma^{2}(\widehat{w}) \approx \frac{\sigma^{2}(N)}{q}
$$

Equation (112) can be used to estimate the minimum number of "wind-off" data points needed to satisfy a model weight prediction tolerance $T O L(\widehat{w})$ that a wind tunnel customer may specify. The corresponding relationship can be obtained by first taking the square root on both sides of Eq. (112). Then, we get:

$$
\sigma(\widehat{w}) \approx \frac{\sigma(N)}{\sqrt{q}}
$$

By inspection, the following inequality has to be satisfied in order to meet a customer's tolerance for the model weight prediction:

$$
\frac{\sigma(N)}{\sqrt{q}}<\operatorname{TOL}(\widehat{w})
$$

Finally, after solving inequality (114) for the total number of "wind-off" data points, we get the relationship:

\section{MINIMUM NUMBER OF "WIND-OFF" DATA POINTS}

$$
q>\frac{\sigma^{2}(N)}{T O L^{2}(\widehat{w})}
$$

Inequality (115) clearly shows that the minimum number of "wind-off" data points needed to meet a customer's tolerance is directly proportional to the variance in the normal force measurement. This variance in the normal force measurement is not simply the square of the standard deviation of the back-computed normal force residuals that are obtained after completion of a balance calibration data analysis. It also includes errors associated with test procedures, instrumentation, and hardware that are used to obtain the "wind-off" data points in the test section of the wind tunnel. 


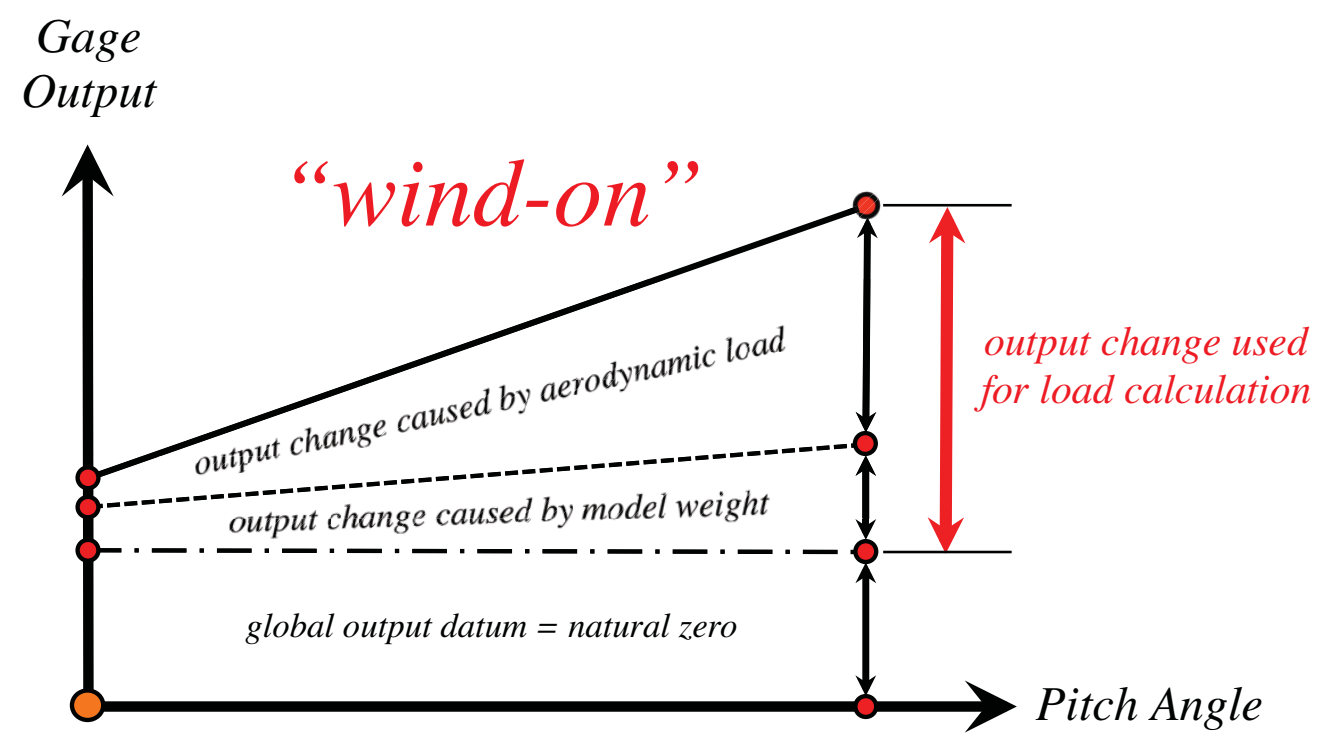

Fig. 1 Balance gage output parts of a "wind-on" data point.

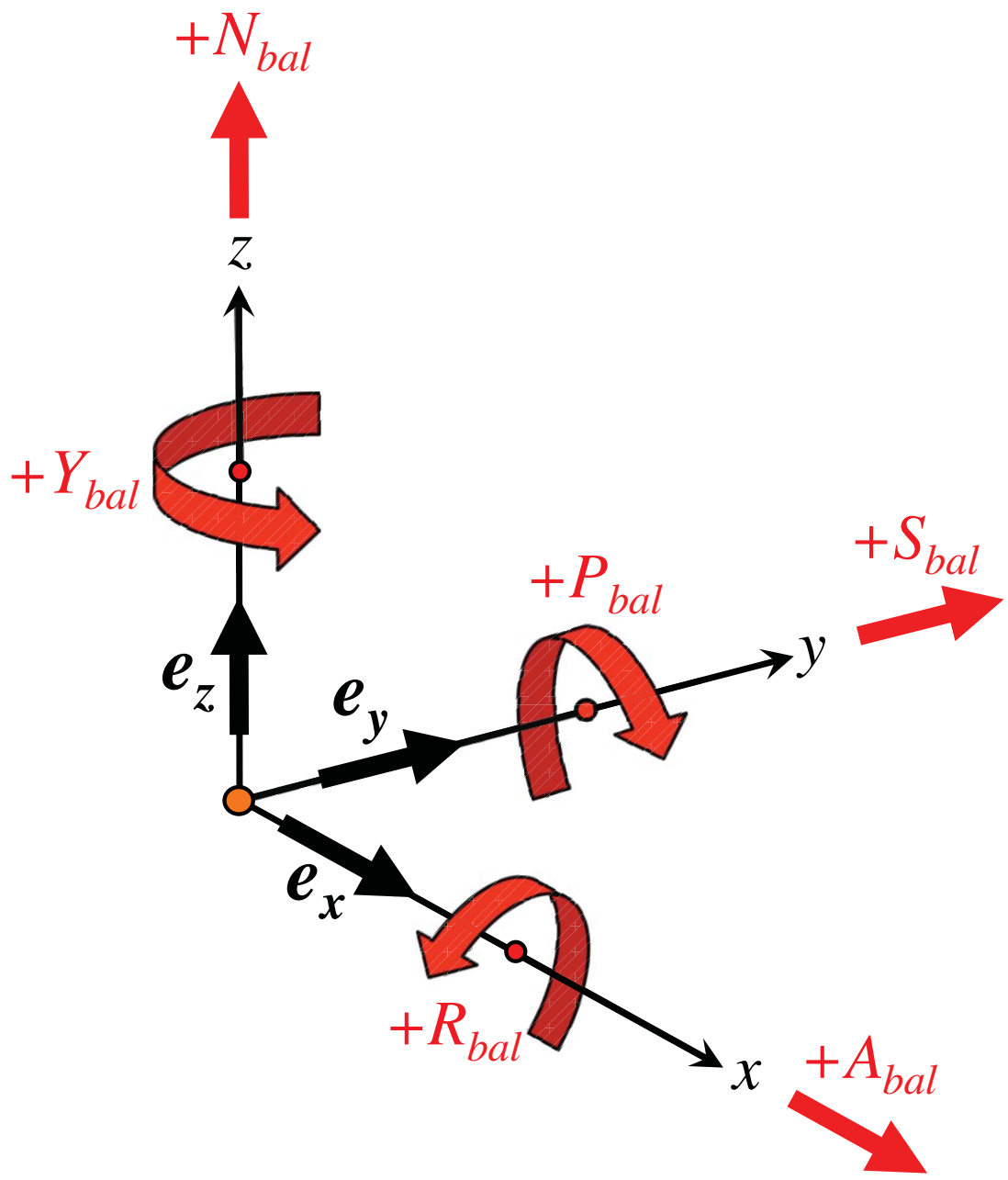

Fig. 2 Definition of Cartesian coordinates and load signs in the "primary" balance axis system. 


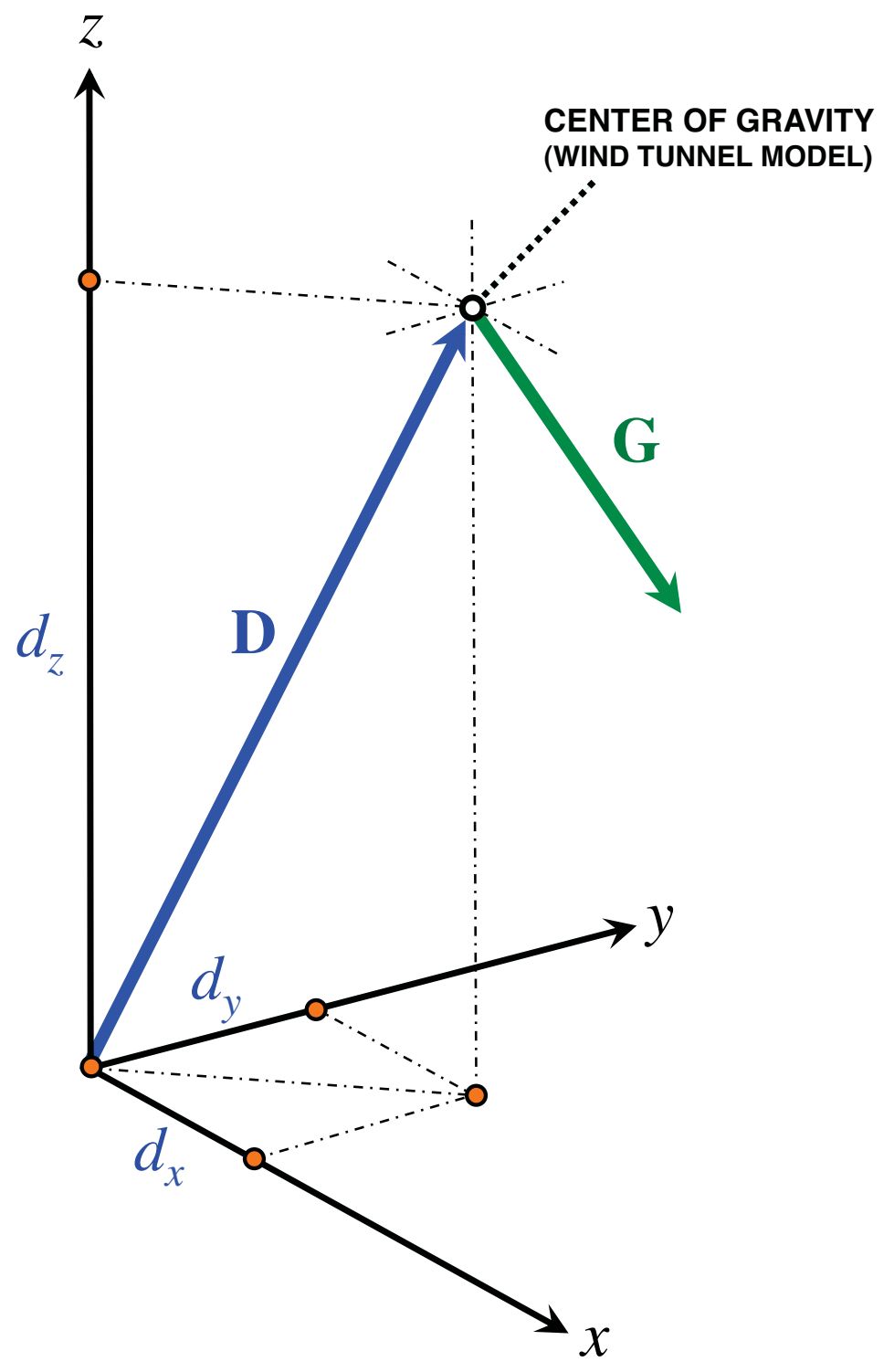

Fig. 3 Description of the gravitational acceleration and the center of gravity coordinates of a wind tunnel model in the "primary" balance axis system. 


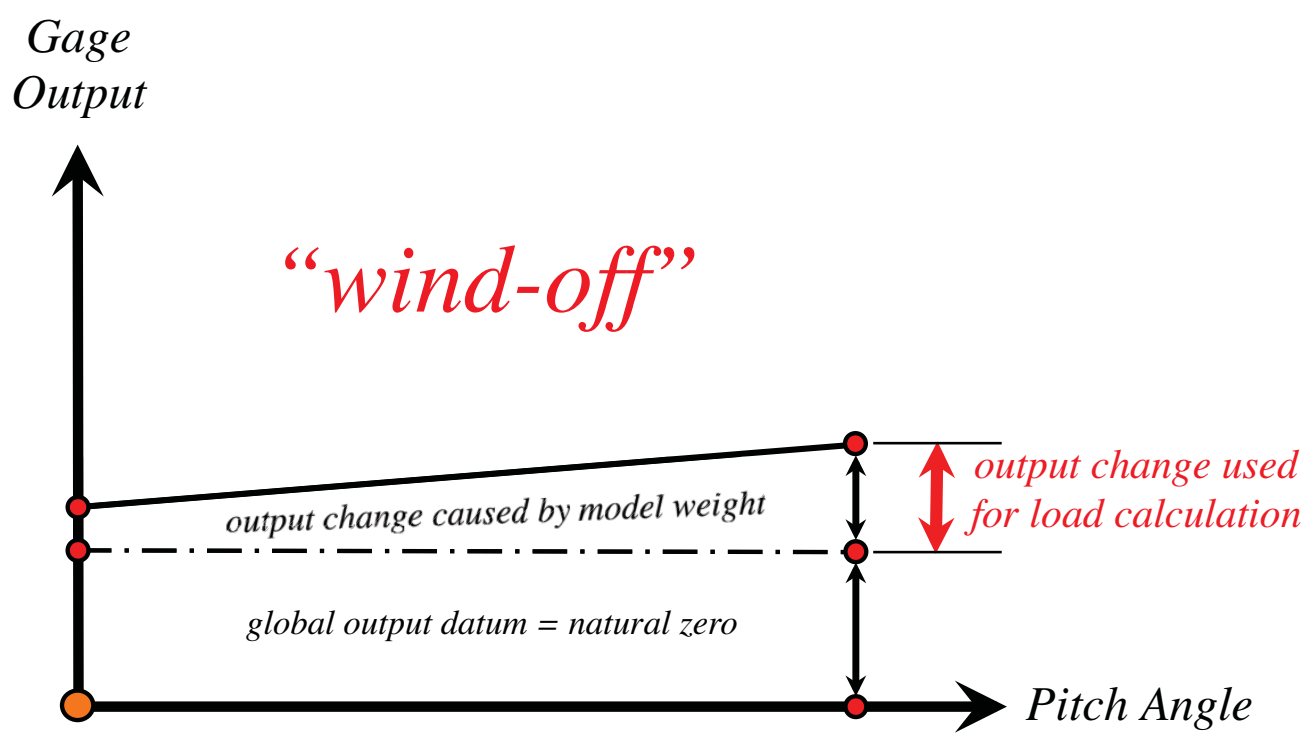

Fig. 4 Balance gage output parts of a "wind-off" data point.

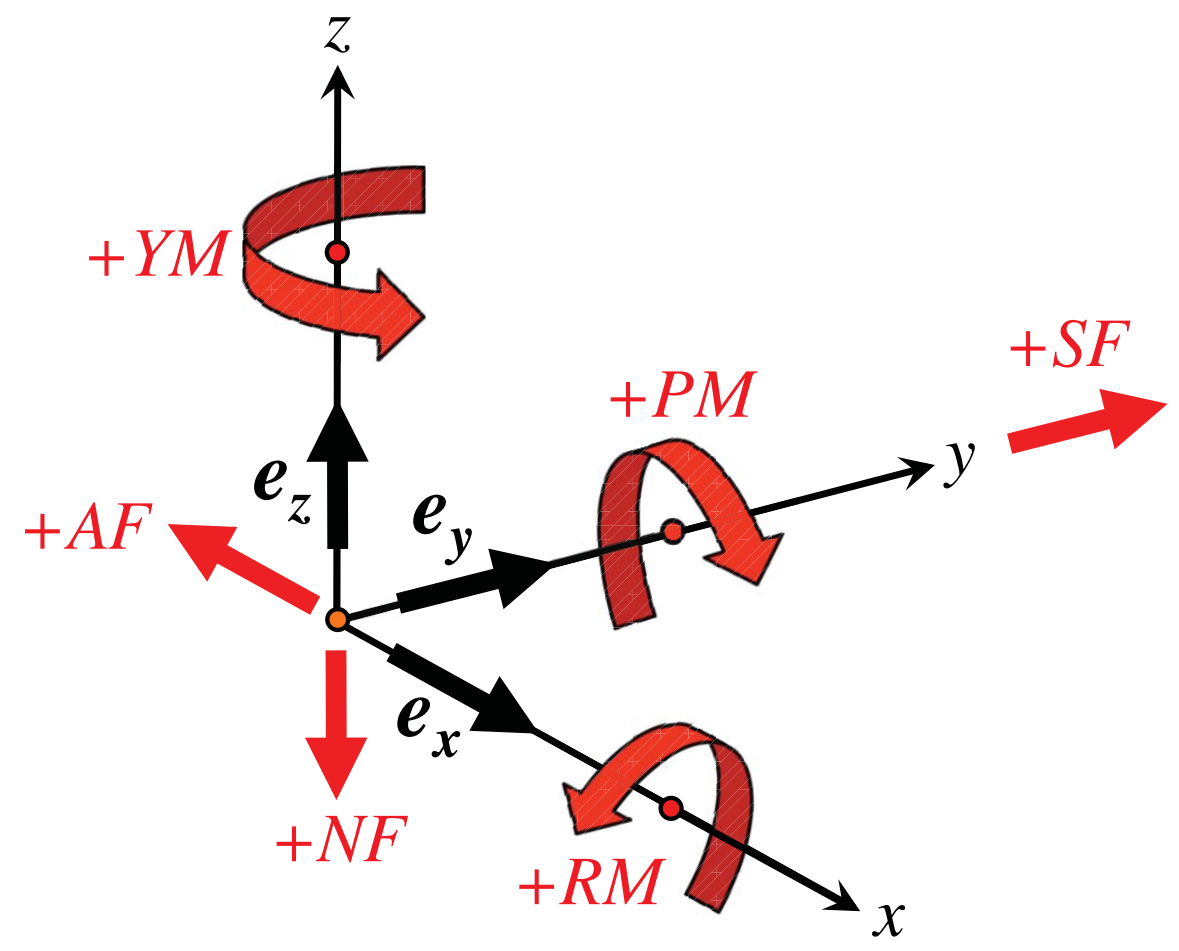

Fig. 5 Definition of Cartesian coordinates and load signs in the "alternate" balance axis system (the definition of the coordinates and load signs is copied from Ref. [1], p. 3, Fig. 1). 


\begin{tabular}{|c|c|c|}
\hline "Wind-On" Test Condition & "Wind-Off" Test Condition & Comments \\
\hline $\begin{array}{l}\text { Balance Loads of a } \\
\text { Single Data Point } \\
A_{b a l}, S_{b a l}, \ldots, Y_{b a l}\end{array}$ & $\begin{array}{c}\text { Balance Loads of Point Set } \\
\begin{array}{c}A_{b a l}(\xi), S_{b a l}(\xi), \ldots, Y_{b a l}(\xi) \\
\text { where } \\
1 \leq \xi \leq q\end{array}\end{array}$ & $\begin{array}{l}\text { Loads are obtained after the appli- } \\
\text { cation of the load iteration process } \\
\text { that uses (i) the difference between } \\
\text { raw outputs and natural zeros and } \\
\text { (ii) the data reduction matrix as } \\
\text { input; "wind-on" and "wind-off" } \\
\text { loads are computed using the same } \\
\text { natural zero set for the iterations. }\end{array}$ \\
\hline $\begin{array}{l}\text { Aerodynamic Loads } \\
\text { of a Single Data Point } \\
\begin{array}{c}A_{\text {aero }}=A_{b a l}-A_{\text {weight }} \\
S_{\text {aero }}=S_{b a l}-S_{\text {weight }} \\
\quad \ldots\end{array} \\
Y_{\text {aero }}=Y_{b a l}-Y_{\text {weight }}\end{array}$ & $\begin{array}{l}\begin{array}{c}\text { Aerodynamic Loads } \\
\text { of Point Set } \\
A_{\text {aero }}(\xi)=0 \\
S_{\text {aero }}(\xi)=0 \\
\cdots \\
Y_{\text {aero }}(\xi)=0\end{array}\end{array}$ & $\begin{array}{l}\text { The aerodynamic loads on the model } \\
\text { in the "wind-on" test condition } \\
\text { are a primary result of the wind } \\
\text { tunnel test. }\end{array}$ \\
\hline $\begin{array}{l}\text { Unit Gravity Vector } \\
\text { Components of a } \\
\text { Single Data Point } \\
\quad \gamma_{x}, \gamma_{y}, \gamma_{z}\end{array}$ & $\begin{array}{c}\text { Unit Gravity Vector } \\
\text { Components of Point Set } \\
\gamma_{x}(\xi), \gamma_{y}(\xi), \gamma_{z}(\xi) ; 1 \leq \xi \leq q\end{array}$ & $\begin{array}{l}\text { Vector components are the result } \\
\text { of model attitude measurements. }\end{array}$ \\
\hline $\begin{array}{c}\text { Forces caused by } \\
\text { the Model Weight } \\
A_{\text {weight }}=\gamma_{x} \cdot \widehat{w} \\
S_{\text {weight }}=\gamma_{y} \cdot \widehat{w} \\
N_{\text {weight }}=\gamma_{z} \cdot \widehat{w} \\
\text { Moments caused by } \\
\text { the Model Weight } \\
R_{\text {weight }}=\gamma_{z} \cdot \widehat{w} \cdot \widehat{d}_{y}-\gamma_{y} \cdot \widehat{w} \cdot \widehat{d}_{z} \\
P_{\text {weight }}=\gamma_{x} \cdot \widehat{w} \cdot \widehat{d}_{z}-\gamma_{z} \cdot \widehat{w} \cdot \widehat{d}_{x} \\
Y_{\text {weight }}=\gamma_{y} \cdot \widehat{w} \cdot \widehat{d}_{x}-\gamma_{x} \cdot \widehat{w} \cdot \widehat{d}_{y}\end{array}$ & 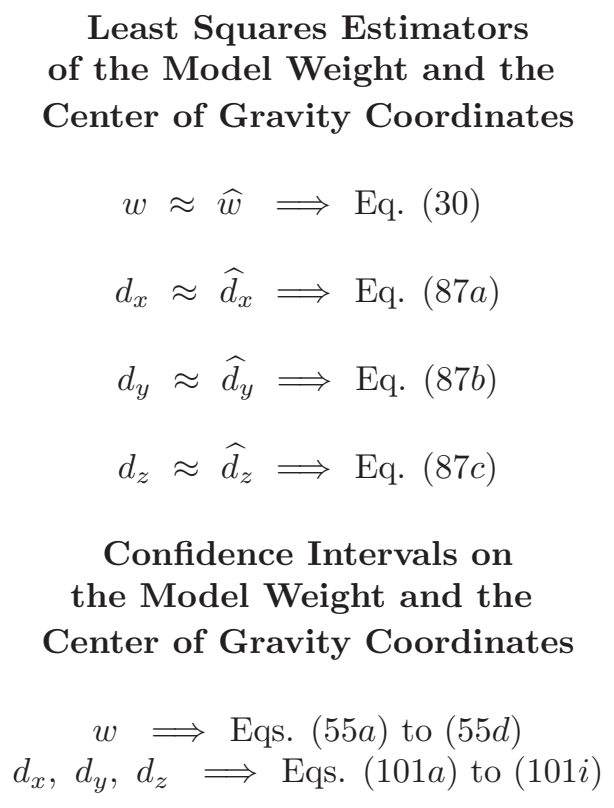 & $\begin{array}{l}\text { The least squares estimators of } \\
\text { the model weight and the center } \\
\text { of gravity coordinates, i.e., } \widehat{w}, \widehat{d}_{x} \text {, } \\
\widehat{d}_{y}, \widehat{d}_{z} \text {, and the unit gravity vector } \\
\text { components of the "wind-on" test } \\
\text { condition, i.e., } \gamma_{x}, \gamma_{y}, \gamma_{z} \text {, are used } \\
\text { as input for the calculation of } \\
\text { forces and moments associated with } \\
\text { the model weight; the least squares } \\
\text { estimators are constant as long as } \\
\text { the weight distribution and the } \\
\text { total model weight do not change. }\end{array}$ \\
\hline
\end{tabular}

Fig. 6 Summary of basic elements of the new method to predict model weight corrections. 